\title{
DEVELOPMENT OF A HUMAN TIBIOFEMORAL JOINT FINITE ELEMENT MODEL TO INVESTIGATE THE EFFECTS OF OBESITY AND MALALIGNMENT ON JOINT CONTACT PRESSURE
}

\author{
A Thesis \\ presented to \\ the Faculty of California Polytechnic State University, \\ San Luis Obispo \\ In Partial Fulfillment \\ of the Requirements for the Degree \\ Master of Science in Mechanical Engineering
}

by

Meghan Sylvia

June 2015 


\section{(C) 2015}

Meghan Sylvia

ALL RIGHTS RESERVED 
TITLE:

AUTHOR:

DATE SUBMITTED:

COMMITTEE CHAIR:

COMMITTEE MEMBER:

COMMITTEE MEMBER:
Development of a Human Tibiofemoral Joint

Finite Element Model to Investigate the Effects of Obesity and Malalignment on Joint Contact Pressure

Meghan Sylvia

June 2015

Stephen Klisch, Ph.D.

Adjunct of Mechanical Engineering

Scott Hazelwood, Ph.D.

Professor of Biomedical Engineering

Joseph Mello, Ph.D.

Adjunct of Mechanical Engineering 


\section{ABSTRACT}

Development of a Human Tibiofemoral Joint Finite Element Model to Investigate the Effects of Obesity and Malalignment on Joint Contact Pressure

Meghan Sylvia

Obesity is a known risk factor for osteoarthritis $(O A)$. Excess body weight generates greater joint contact forces at the knee; however, obese individuals alter their gait to decrease joint contact forces. Knee malalignment has been identified as a strong mediating factor between obesity and knee $O A$ progression. Excess body weight acting on a varus malaligned knee would have an additive effect on cartilage stress and could cause stress levels to exceed the threshold limit for damage and loss of cartilage matrix.

A finite element (FE) model of the human tibiofemoral joint was developed and validated in order to investigate changes in cartilage pressure due to obesity and knee varus malalignment. The results of this analysis show that obese loading conditions caused greater contact pressure in both the lateral and medial tibiofemoral compartments at most phases of stance. Increased contact pressure applied cyclically during daily activities could make obese individuals more susceptible to OA. Varus malalignment increased medial contact pressure as expected, but lateral contact pressure also increased during midstance for both normal weight and obese load conditions. These results suggest that varus malaligned individuals could be susceptible to OA development in both tibiofemoral compartments due to the overall increase in joint contact pressure.

As a qualitative tool, the FE model functioned well in highlighting changes in joint contact pressure due to the addition of obesity or varus malalignment. Further work can be done to increase confidence in the quantitative outputs of the model by using more sophisticated material models for soft tissue structures and incorporating the patellofemoral joint into the FE model.

Keywords: Osteoarthritis, finite element, human tibiofemoral joint, obesity, knee malalignment, articular cartilage, contact pressure 


\section{ACKNOWLEDGMENTS}

Thanks to my advisors, Dr. Stephen Klisch and Dr. Scott Hazelwood, for their guidance and support in the completion of this thesis. Additional thanks to Dr. Saikat Pal and Dr. Joseph Mello for their helpful input through the course of this project.

Special thanks to Zach Lerner and Dr. Ray Browning for their collaboration and willingness to answer my many questions. Additional thanks to Dr. Otto Schueckler and Dr. David Tuttle for their assistance interpreting MRIs.

A very big thanks to Nick Czapla for his friendship and partnership on this project.

Finally, thank you to my family and friends for their unconditional love and support. 
LIST OF TABLES .................................................................................. vii

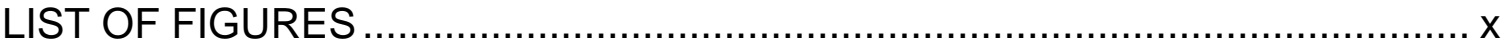

CHAPTER

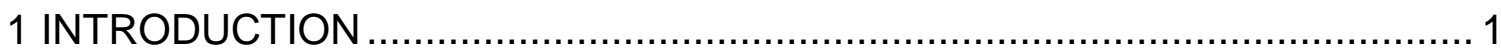

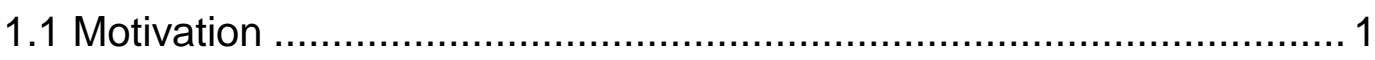

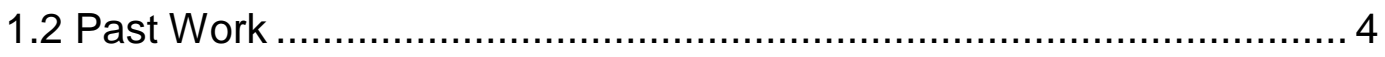

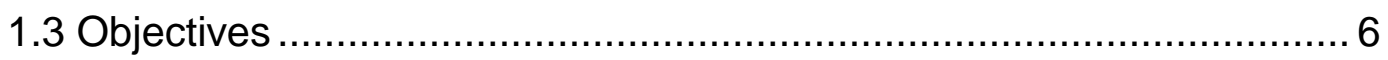

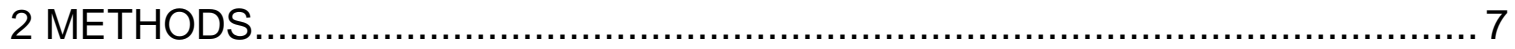

2.1 Solid Model Development ......................................................... 7

2.1.1 Subject Information and MRI Settings ............................... 7

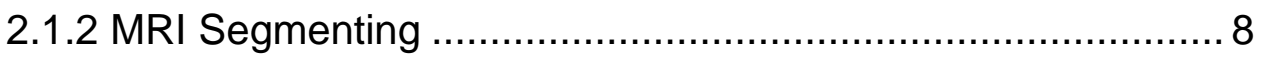

2.1.3 Surface Processing ........................................................ 10

2.1.4 Solidworks Generated Models ......................................... 13

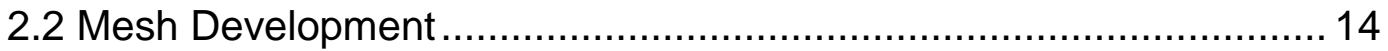

2.2.1 Partitioning …….......................................................... 14

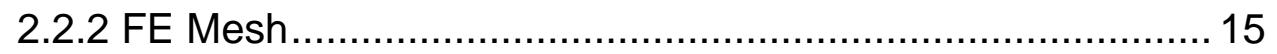

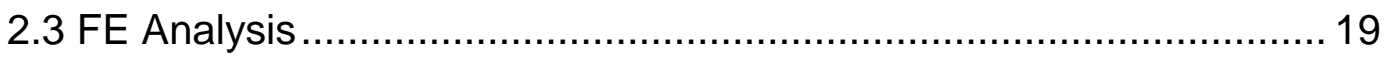

2.3.1 Material Assignment....................................................... 20

2.3.2 Interactions and ConstraintS ............................................. 22

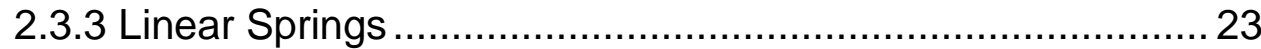

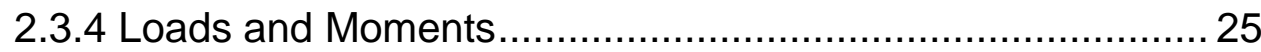

2.3.5 Boundary Conditions ..................................................... 27

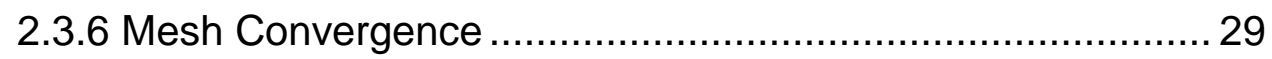

2.3.7 Damping Factor Stabilization ........................................... 32

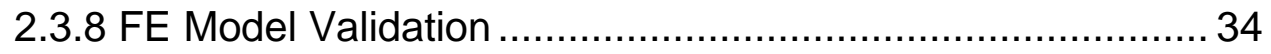

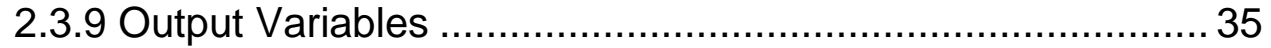

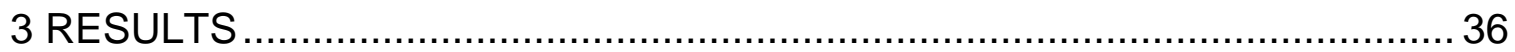

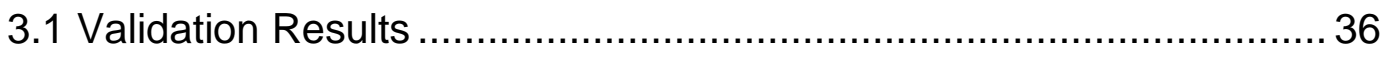

3.1.1 Maximum Contact Pressure Validation .............................. 36 


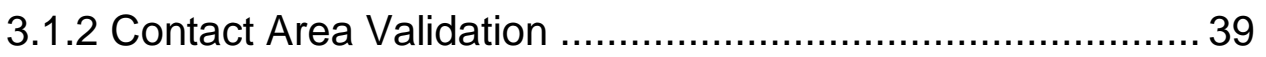

3.2 Gait Analysis Results ................................................................ 42

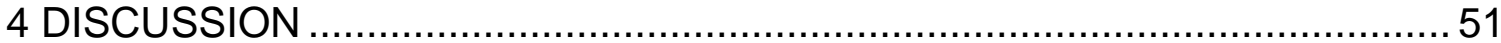

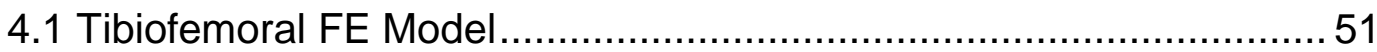

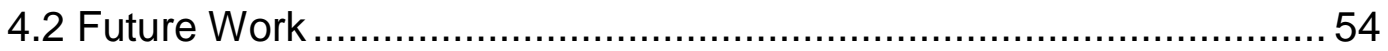

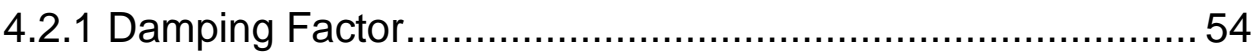

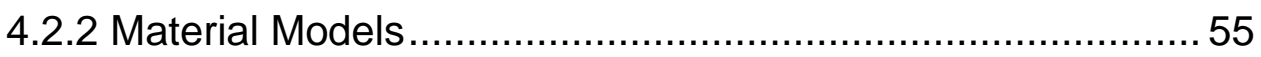

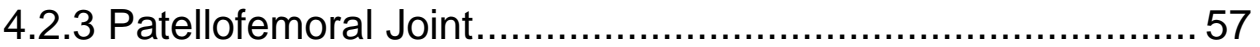

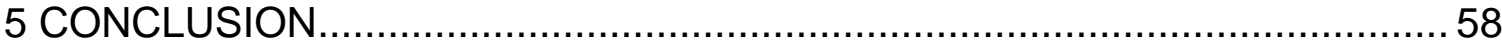

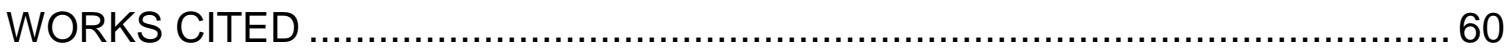

APPENDICES

Appendix A: Contour Plots of Contact Pressure on the Surface of the Tibial and Femoral Articular Cartilages 


\section{LIST OF TABLES}

Table

1: Anatomical Dimensions of the LCL and MCL [26-31].

2: Element type, material type, and material properties assigned to each

solid model geometry.

3: Spring Stiffness constants assigned to the anterior and posterior horns

of the menisci and the distal face of the LCL. The springs were

either defined between two nodes in a line of action or in the

Abaqus coordinate system

4: The gender and body weight of the obese and normal weight gait analysis participants.

5: Summary of the flexion angles and loads for a normal weight individual at each of the 6 stages of stance analyzed in this study. Forces and moments are defined based on the local Abaqus coordinate system described above.

6: Summary of the flexion angles and loads for an obese individual at each of the 6 stages of stance analyzed in this study. Forces and moments are defined based on the local Abaqus coordinate system described above.

7: Summary of the loads implemented during the convergence study. 29

8: The ratio of viscous force to total forces calculated for each subject type across all investigated phases of stance. 
9: The ratio of viscous damping energy to total strain energy calculated for each subject type across all investigated phases of stance. 33

10: A summary of the maximum contact pressure validation analysis results. The maximum contact pressure determined from the FE model is compared to the maximum contact pressure values obtained from experimental cadaver studies.

11: A summary of the contact area validation analysis results. The contact area determined from the FE model is compared to the contact area values obtained from experimental cadaver studies. 42

12: The maximum contact pressure on the surface of the femoral cartilage as predicted by the tibiofemoral FE model. The location of the maximum contact pressure is indicated as either the medial or lateral femoral condyle. CPress $=$ contact pressure, $\mathrm{HS}=$ heel strike, and TO = toe off. 46

13: Contact areas at the surface of the medial, lateral, and femoral articular cartilage for each subject type across all investigated phases of stance. 


\section{LIST OF FIGURES}

Figure

1: An example of the anatomical mask of the tibia. Masks such as the one above were created for each individual anatomical structure. 9

2: Superior and side view of the $(A)$ lateral tibial cartilage STL mesh file generated in Mimics, (B) the lateral tibial cartilage mesh after editing in Solidwork's mesh prep wizard, and (C) the lateral tibial cartilage solid model after surface editing in Solidworks. Notice that the irregular, curved edges of the STL mesh in (A) are replaced by flat, even faces in (B) and (C).

3: Sketches were projected onto the femur solid model creating the curves shown in blue above. The curved lines define the partitions used for meshing.

4: The computation block mesh $(A)$ is defined in Truegrid to match the partitioned sections created on the solid model. The partitions can be seen in (B) as curves defining the edges of the geometry. The computational blocks in $(\mathrm{A})$ are projected to the curves and surfaces which produces a rough mesh in (C). The mesh is refined in (D) by increasing the mesh density.

5: FE mesh of the tibiofemoral joint with anatomical structures labeled.

The assembled FE model consists of the proximal tibia, distal femur, articular cartilage (femoral, and medial and lateral tibial), 
menisci (medial and lateral), and ligaments $(\mathrm{ACL}=$ anterior cruciate ligament, $\mathrm{PCL}=$ posterior cruciate ligament, $\mathrm{LCL}=$ lateral collateral ligament, $\mathrm{MCL}=$ medial collateral ligament).

6: Spring elements of each meniscus horn are indicated in pink.

7: The location of the nodes analyzed in the mesh convergence study are indicated in red.

8: Convergence study results shown as a plot of contact pressure at the indicated convergence node vs the degrees of freedom of the mesh. Selected mesh degrees of freedom are indicated by the gray shaded markers.

9: The bar graph of maximum contact pressure on the surface of the medial tibial cartilage as predicted by the tibiofemoral FE model. Maximum contact pressure is displayed for each subject type across all investigated phases of stance. $\mathrm{HS}=$ heel strike and $\mathrm{TO}=$ toe off.

10: The bar graph of maximum contact pressure on the surface of the lateral tibial cartilage as predicted by the tibiofemoral FE model. Maximum contact pressure is displayed for each subject type across all investigated phases of stance. $\mathrm{HS}=$ heel strike and $\mathrm{TO}=$ toe off.

11: Contact pressure in MPa on the superior surface of the lateral and medial tibial cartilage and the inferior aspect of the femoral cartilage 
for each subject type at $25 \%$ stance. $(A=$ anterior and $\mathrm{P}=$ posterior

direction)

12: The plot of medial and later tibial cartilage contact pressure as a

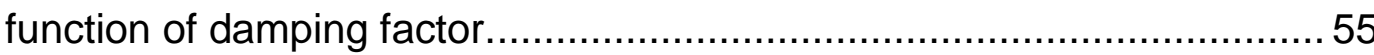




\section{CHAPTER 1}

\section{INTRODUCTION}

\subsection{Motivation}

Osteoarthritis $(\mathrm{OA})$ is a degenerative condition characterized by the breakdown and eventual loss of joint articular cartilage. OA is the most common form of arthritis, affecting a reported 27 million Americans in 2006; a number that is only expected to increase with the aging population [1]. On a global scale, OA is estimated to be the fourth leading cause of disability [2]. Of those affected by OA, $80 \%$ experience limitations in movement and $25 \%$ are unable to perform major daily activities [1], [3]. From a financial perspective, OA imposes a significant burden due to the direct cost of medical intervention as well as the indirect cost of loss of work-place productivity [2]. A 2007 study determined that OA and other arthritis-related conditions cost the U.S. economy nearly $\$ 128$ billion per year [1]. On an individual level, a person suffering from OA can expect to pay approximately $\$ 5700$ per year due to medical costs and other expenses [1].

An increased understanding of the pathogenesis and progression of $O A$ would have advantageous socio-economic ramifications. While the cause of OA is not precisely known, identified risk factors for OA include advanced age, obesity, female gender, joint injury, and genetic predisposition [1-3]. The healing capacity of articular cartilage is minimal due to its poor vascularity and current pharmaceutical options for OA patients are limited to palliative care [1], [4]. In the case where pain is severe and mobility is drastically hindered, joint replacement 
surgery may be an option. In the US, the total number of hip and knee joint replacements exceeds 350,000 annually [5].

Although OA predominately affects the joints at the knee, hip, hand, spine, and ankle, the greatest disability burden is attributed to OA at the hip and knee [1], [3], [5]. Significant effort and research has gone towards understanding knee OA due to its higher rate of incidence [5], [6]. The relationship between knee OA and obesity is of particular interest for several reasons. First, obesity is most easily modified of the risk factors for knee OA listed above [7], [8]. Second, obesity is more strongly linked to OA at the knee than at other lower extremity joints [7-9]. Third, obesity has been proven to be related to both the development and progression of knee OA [7], [9], [10] .

From a biomechanical perspective, excessive body weight alone does not explain the relationship between obesity and knee OA. Although increased body weight generates greater mechanical loads at the knee, obese individuals may develop gait modifications to address the excess joint loading. Obese adults tend to have a shorter stride length, slower walking speed, and increased stance and double support phases of gait [11], [12]. Reduced walking speed in particular has been shown to decrease ground reaction forces and moments, which in turn would decrease loading at the knee [11]. In addition, healthy cartilage responds positively to increasing load and can become conditioned to greater cyclic loading [13], [14]. A more plausible explanation is that the relationship between obesity and knee OA is multifaceted, with additional factors influencing the mechanical impact of excess weight at the knee. 
Knee malalignment is recognized as a strong mediating factor between obesity and knee OA [9]. Alignment is determined by the angle formed between the mechanical axes of the femur and the tibia. Malalignment alters cartilage stress by offsetting the knee from its natural loading pattern and causing irregular load distributions within the tibiofemoral compartment [15]. Varus ('bowlegged') alignment redirects body weight medially and increases loading in the medial tibiofemoral compartment. Valgus ('knock-kneed') alignment redirects weight laterally and increases loading in the lateral tibiofemoral compartment [10], [15] [16].

In a neutrally aligned knee, the medial tibiofemoral compartment supports 2.5 times more load during gait than the lateral compartment [10], [17]. Even a small incremental change towards varus alignment would have the potential to increase compressive loading to pathological levels [6], [10]. Excess body weight acting on a varus aligned knee would have an additive effect on cartilage stress and could potentially cause stress levels to exceed the threshold limit for damage to, and subsequent loss of, cartilage matrix. In contrast, valgus alignment creates a more equitable distribution of loading across the tibiofemoral compartment. It would require severe valgus alignment before load was disproportionally born by the lateral compartment [10]. These factors help account for the fact that the medial tibiofemoral compartment is 10 times more likely to be affected by $\mathrm{OA}$ [17].

An accurate understanding of cartilage stress is important for knee OA treatment and prevention. Weight loss is known to improve pain and function in 
obese patients with knee OA, but the process of losing weight often involves activities that are high impact on the knee [4], [7]. A comprehensive knee model that can predict cartilage loading for different exercises and body types may facilitate the analysis of motions and exercises that minimize cartilage stress.

\subsection{Past Work}

The interaction between varus malalignment and obesity in relation to knee OA has been analyzed in detail over the past decade, but research has been limited to quantitative gait analysis. Previous studies have used kinetic and kinematic data from obese participants to compute the external knee adduction moment (KAM) as a proxy for knee joint load. Although the external KAM is a valid indicator of load on the tibiofemoral compartment, it neglects the contribution of soft tissue and muscles to internal joint loading [6]. Additionally, the external KAM is primarily an indication of absolute load and does not provide information regarding the stress distribution throughout the tibiofemoral compartments.

Recent advances in musculoskeletal modeling software have enabled researchers to produce more robust assessments of joint loading. Programs such as OpenSim (simtk.org, Stanford, CA) use 3D motion data to create dynamic simulations of human movement which can then be used to calculate muscle and force components of the internal joint load. Richards et al. [18] applied lower extremity musculoskeletal modeling in OpenSim to investigate knee contact force and muscle activation in patients with varying levels of $O A$ severity. Along these same lines, Haight et al. and Lerner et al. [19], [20] both 
utilized OpenSim's modeling capabilities to determine knee contact forces and muscle forces in obese adults while walking. A review of the literature suggests that there has been no attempt to use musculoskeletal modeling to determine the combined effects of knee malalignment and obesity in relation to $O A$ development.

Finite element analysis (FEA) has been used to develop a greater comprehensive understanding of the effects of loading on soft tissue stress and strain within the tibiofemoral joint. FEA has the distinct advantage over both gait analysis and musculoskeletal modeling in that it can both quantify and predict distributions of stress and strain in each anatomical structure of the knee. Detailed 3D finite element (FE) models of the tibiofemoral joint have been developed with varying levels of complexity over the past two decades. One such model created by Shirazi Adl et al. from the CT scans of a healthy human knee has been adapted to study the effects of various biomechanical factors on articular cartilage contact pressure. By altering the material properties of the articular cartilage, Shirazi-Adl et al. [21], [22] have shown that osteochondral defects and cartilage deterioration produced changes in pattern and magnitude of articular cartilage contact pressure. Pena et al. [23] developed a similar tibiofemoral FE model to investigate viscoelastic material models of biological soft tissue. Their work ultimately led to a validated constitutive model that produced realistic predictions of stress and strain in ligaments and collagen tissue. Although FE models of the human knee have become more sophisticated over time and have been utilized in many different capacities in the study of OA, 
the research presented in this thesis is the first attempt to determine the combined effects of obesity and knee malalignment on articular cartilage stress.

\subsection{Objectives}

The long-term goal of this project is to apply the FE model in a clinical application in order to determine patient-specific exercises that minimize knee joint loading for subjects at high risk for knee OA. Future studies will combine motion analysis during walking and biking with a subject-specific FE model to produce individualized treatment and prevention plans.

The specific objectives of this thesis are to develop and validate a tibiofemoral joint FE model and to predict cartilage stress in response to varying levels of body weight and knee alignment during walking. The tibiofemoral FE model will be used to test the following hypotheses: 1) in a neutrally aligned knee, increased mechanical loading due to excess body weight will not cause articular cartilage stress to exceed pathological levels for cell death and cartilage surface damage; and 2) varus malalignment will amplify the effect of excess body weight and cause greater levels of articular cartilage stress in the medial tibiofemoral compartment, but not in the lateral tibiofemoral compartment. 


\section{CHAPTER 2}

\section{METHODS}

\subsection{Solid Model Development}

The following subsections describe the creation of the tibiofemoral solid model using magnetic resonance images (MRIs) of a healthy, non-arthritic knee.

\subsubsection{Subject Information and MRI Settings}

MRIs were obtained from a 33 year old male with no known knee conditions. The subject had a body mass index (BMI) of 26.5 , placing him in at the lower end of the overweight spectrum. BMI is a calculation of an individual's weight in relation to height and is defined as:

$$
\text { BMI }=\frac{\text { mass }(\mathrm{kg})}{\text { height }^{2}\left(\mathrm{~m}^{2}\right)}
$$

BMI is a notoriously imprecise measure of health and does not account for a person's fat to muscle ratio. Given that the subject was an avid runner, it is likely that his percentage of lean muscle is augmenting the BMI calculation. Therefore, there is nothing to suggest that the subject was especially unhealthy or that he possessed an excess of body fat.

Alignment of the subject's knee was determined by measuring his $Q$ angle while standing with a long arm goniometer. Results from this measurement indicate a $Q$ angle of $11^{\circ}$, which places the subject in the acceptable $Q$ angle range for males and confirms normal knee alignment [24].

The subject's knee was imaged using a GE Medical Systems MRI at Stanford University's Lucas Imaging Center. Images were captured on the 
sagittal plane at $1.5 \mathrm{~mm}$ intervals. The MRIs were fat suppressed with a gradient echo sequence and an in-plane resolution of $0.3516 \mathrm{~mm}$.

\subsubsection{MRI Segmenting}

Mimics (Materialise, NV, Leuven, Belgium) was used to segment the MRIs and construct 3D geometry of each individual bone and soft tissue structure. The bones included in the model are the distal head of the femur and the proximal head of the tibia. The model also includes the four main stabilizing ligaments of the knee: the medial collateral ligament (MCL), lateral collateral ligament (LCL), anterior cruciate ligament $(A C L)$, and the posterior cruciate ligament $(P C L)$. Finally, the medial and lateral menisci and the articulating cartilage of the tibiofemoral joint are included in the model.

MRI segmentation is a process through which anatomical structures are partitioned on a digital image by assigning a label to specific pixels. Mimics uses a method referred to as "thresholding" to select pixels that fall within a userdefined region of the gray-scale. By utilizing the threshold function, the user can create a "mask" to identify boundaries or regions of interest, such as the mask of the tibia shown in Figure 1. Threshold profiles for bone and soft tissue were defined to minimize the amount of manual segmentation required, but it was not uncommon for multiple structures to possess similar regions of the gray-scale. After thresholding, each mask was edited by using the erase and draw tools to create a distinct boundary around the anatomical structure. Mimics then creates a 3D surface model by stacking the masks drawn on the 2D images. The MCL and LCL could only be definitively identified on a single MRI each, which was an 
insufficient number of masks to produce a 3D geometry. The 3D models for these structures were therefore constructed manually in Solidworks as described in Section 2.1.4.

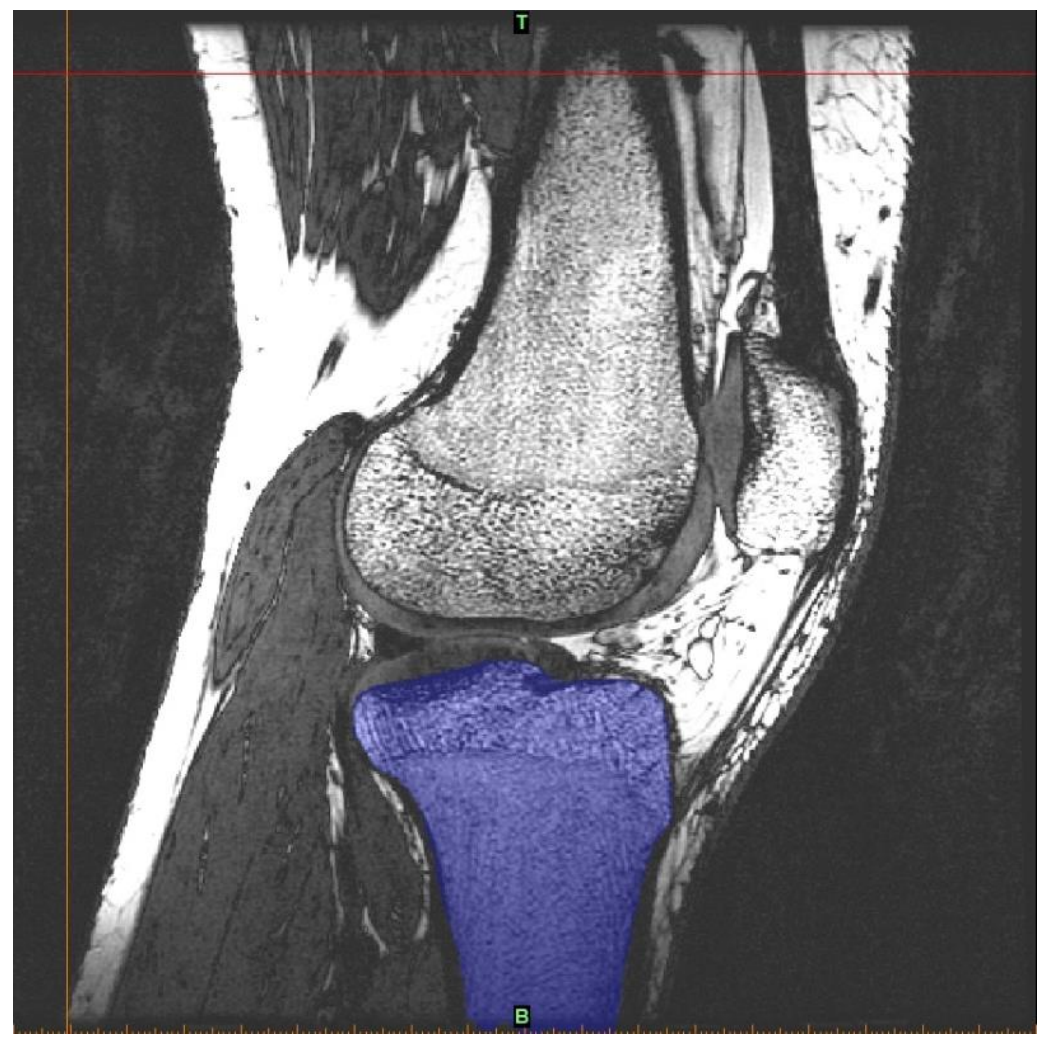

Figure 1: An example of the anatomical mask of the tibia. Masks such as the one above were created for each individual anatomical structure.

The highest quality setting was selected for the 3D model calculation to ensure that accurate models were produced. Segmentation inherently introduces noise in the data, which can produce rough, uneven surfaces on the 3D model. Mimic's Gaussian smoothing algorithm was used to remove irregularities in the surface geometry. A Gaussian smoothing factor of 0.8 was selected after several trials. Large structures, such as the tibia and femur, were smoothed over 500 iterations. Soft tissue structures were smoothed over 200 iterations. 


\subsubsection{Surface Processing}

The smoothed models were exported from Mimics as stereo lithography

(STL) files. STLs are triangulated surface mesh files and are considered ideal for representing anatomical models due to their ability to contour to the geometry [25]. However, triangular surface meshes produce poor solid tetrahedron FE meshes. Therefore, it was necessary to import the STLs into Solidworks (Dassault Systemes, Velizy-Villacoublay, France) for surface processing.

Cartilage and meniscal meshes were first edited using Solidworks' mesh prep wizard application. Data removal tools in the application were used to trim the rounded, tapered edges of the mesh. Next, local smoothing was performed to remove sharp edges that formed as a result of the trimming. These initial steps produced surface meshes with squared-off corners, which ultimately facilitated meshing of the solids. The effects of these edits can be seen by comparing the mesh edges in Figure 2A and 2B.

All structures were then converted into solids as shown in Figure $2 \mathrm{C}$ using Solidworks' automatic surface creation tool. The tool functions by first producing feature lines that follow the curvature of the mesh. Occasionally, the feature lines were manually edited within the surface creation tool interface to better capture the curvature of complex geometries. The boundaries formed by the feature lines define surface regions which are knit together to form a solid. Surface detail was increased iteratively using the surface wizard toolbar until a solid was produced that best captured the mesh geometry. 
A
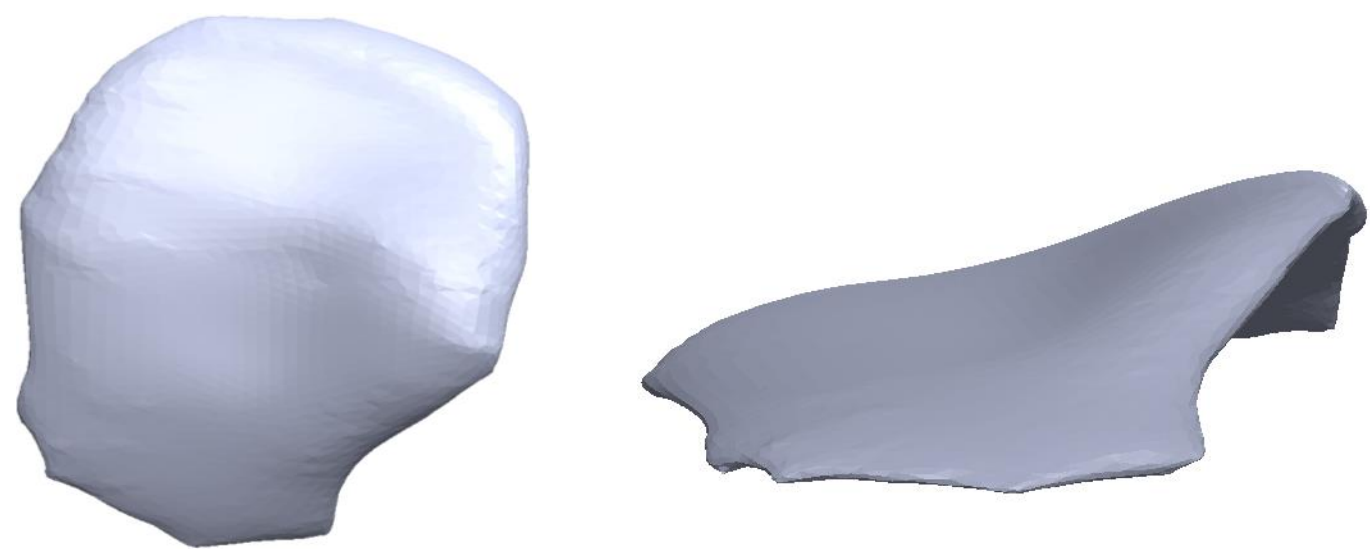

B
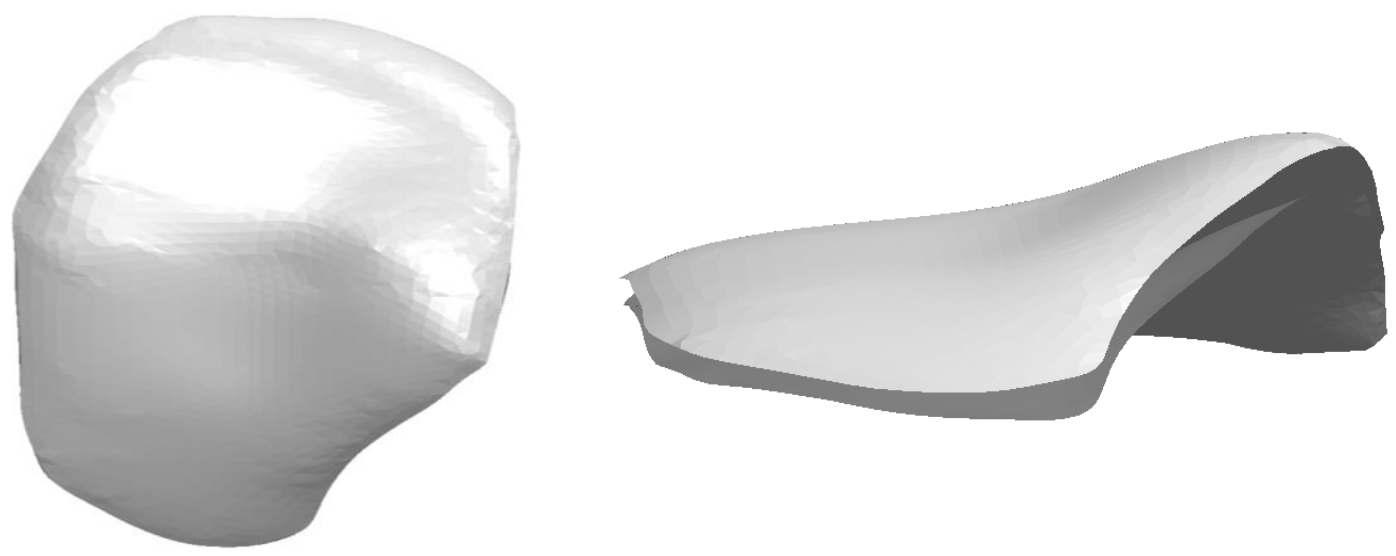

C
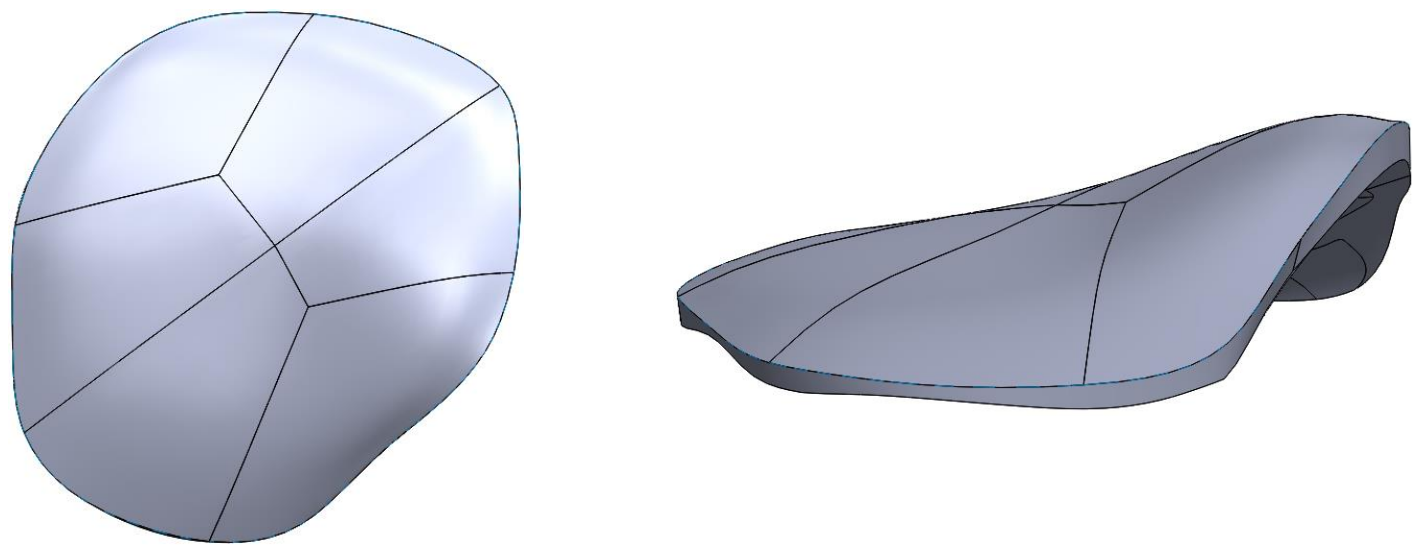

Figure 2: Superior and side view of the $(A)$ lateral tibial cartilage STL mesh file generated in Mimics, $(B)$ the lateral tibial cartilage mesh after editing in Solidwork's mesh prep wizard, and $(C)$ the lateral tibial cartilage solid model after surface editing in Solidworks. Notice that the irregular, curved edges of the STL mesh in (A) are replaced by flat, even faces in $(B)$ and $(C)$. 
Solid parts were assembled in Solidworks based on their relative position in reference to the local coordinate system embedded in the STL files. Upon inspection, it became evident that there was overlap between bone and soft tissue structures. The overlapping regions likely developed as a result of the smoothing iterations performed in Mimics. When Mimics smooths a model, the original volume is preserved, meaning that the volume of the removed regions are redistributed throughout the model. Solidworks' surface cut tool was used to trim overlapping regions. In the case of bone and cartilage overlap, the surface of the bone was used as a guide to cut away from the cartilage surface. Overlap between soft tissue structures was not as prevalent due to the joint space width of the tibiofemoral compartment. Slight overlap between the tibial and femoral cartilage was minimal enough to be assumed negligible.

The assembled tibiofemoral joint model was reviewed at multiple stages by a board certified orthopedic surgeon to ensure that size, shape, and spatial arrangement of the models were anatomically accurate. Based on his professional recommendation, the ACL solid model was edited in Solidworks so that its attachment to the femur was situated more posteriorly on the lateral femoral condyle. Further details regarding changes to the ACL model are described in Section 2.1.4. 


\subsubsection{Solidworks Generated Models}

As previously mentioned, MCL and LCL models could not be generated in Mimics due to the spatial resolution of the MRIs. Solid models of these ligaments were instead created in Solidworks based on anatomical measurements from cadaver studies found in the literature. Cross-sectional areas of the bone attachment sites, ligament volumes, and ligament lengths listed in Table 1 were used to define the LCL and MCL model geometry. Since only the superior half of the LCL is included in the FE model, the cross-sectional area measured at the LCL midsection was used to define distal face of the LCL model.

Table 1: Anatomical Dimensions of the LCL and MCL [26-31].

\begin{tabular}{|c|c|c|}
\hline & MCL & $\mathrm{LCL}$ \\
\hline Length (mm) & 80.73 & 31.51 \\
\hline Volume $\left(\mathrm{mm}^{3}\right)$ & 4592.91 & 227.67 \\
\hline \multicolumn{3}{|l|}{ Femoral Attachment } \\
\hline Cross Sectional Area $\left(\mathrm{mm}^{2}\right)$ & 80.24 & 50.6 \\
\hline Major Axis Half Length ( $\mathrm{mm}$ ) & 6.80 & 4.81 \\
\hline Minor Axis Half Length ( $\mathrm{mm}$ ) & 3.75 & 3.35 \\
\hline \multicolumn{3}{|l|}{ Tibial Attachment / LCL } \\
\hline Distal Face & 270.68 & 7.23 \\
\hline Cross Sectional Area $\left(\mathrm{mm}^{2}\right)$ & 16.34 & 2.13 \\
\hline Major Axis Half Length ( $\mathrm{mm}$ ) & 10.10 & 1.08 \\
\hline Minor Axis Half Length ( $\mathrm{mm}$ ) & & \\
\hline
\end{tabular}

Elliptical sketches were drawn in Solidworks to create the femoral attachments faces of the LCL and MCL. The tibial attachment of the MCL and the distal face of the LCL were was also represented as ellipses. Lofted surfaces were created between the respective $M C L$ and $L C L$ elliptical faces with $3 D$ sketched guide curves added to help define the structure shape and curvature. Finally, the surfaces were knit together in Solidworks to form a solid part. The 
attachment locations of the LCL and MCL were determined based on measurements in published journals [26], [27], [29].

Additional changes were made to the ACL model due to concerns regarding the position of the femoral attachment site. With the guidance of an orthopedic surgeon, The new ACL femoral attachment was made in Solidworks by sketching an ellipse with a major and minor axis half length of $7.01 \mathrm{~mm}$ and $4.23 \mathrm{~mm}$, respectively [32], [33]. A Solidworks sketch of the ACL tibial attachment face was created using the profile of the existing ACL model. A lofted surface was then formed between the ACL attachment faces as described above. The 3D sketched guide curves were edited slightly through an iterative process to produce a volume of $1731.2 \mathrm{~mm}^{3}$ consistent with values found in published studies [32], [33].

The final dimensions and position of the $L C L, M C L$, and ACL models were approved by an orthopedic surgeon before proceeding with mesh generation.

\subsection{Mesh Development}

Solid models were partitioned to facilitate meshing the complex 3D geometry. FE meshes of each structure were generated in TrueGrid Software (XYZ Scientific Applications, Inc.) by projecting block elements onto user defined curves and surfaces. These meshes were subsequently exported to Abaqus (Dassault Systemes) for analysis.

\subsubsection{PARTITIONING}

Each solid model was partitioned in Solidworks by projecting sketches onto the model surface. The projected sketches produced curves that followed 
the contours of the model. Partitions were selected so that the model was divided into 4 sided sections, as can be seen in Figure 3. The number of partitions was determined largely by trial and error; larger, complex models required more partitions while smaller models with simple geometry required fewer. There was particular effort to develop partitioned sections that were equal in size with angles no greater than $135^{\circ}$. Partitioned models were saved as IGES files and exported for meshing.

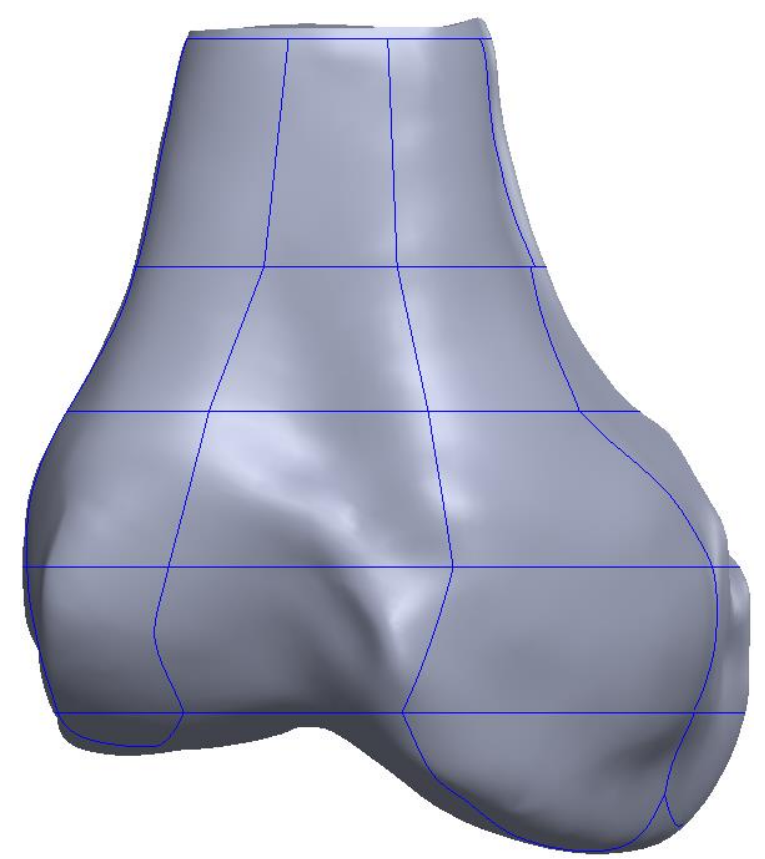

Figure 3: Sketches were projected onto the femur solid model creating the curves shown in blue above. The curved lines define the partitions used for meshing.

\subsubsection{FE Mesh}

Truegrid is a multipurpose tool that allows the user to create a geometry, generate a mesh of the geometry, and assign conditions to the mesh needed for pre-processing. For this purpose of this thesis, only the mesh generation feature of Truegrid was utilized. 
The partitioned models were individually opened as IGES files in Truegrid. The geometry of each model could be viewed as an assemblage of numbered curves and surfaces, where the curves defined the edges of the geometry and the surfaces encompass the area in between the curve-defined regions. The partition lines created in Solidworks were visible as intersecting curves on the faces of the solid models.

Meshes were created using a multi-block projection approach. First, the number of single block mesh elements needed for meshing was specified using the block command. In general, one block mesh was needed for every partitioned section of a given model. Specific arrangements of blocks were created by specifying the number of blocks in each row and column or by deleting individual blocks with the del command. The end result is an array of blocks that represents that geometry of the model, with each block mirroring a partitioned section. An example of an edited block mesh is shown below in Figure 4A.

Next, the edges of each block were projected to the model by calling out the appropriate curve in the curd command, as depicted in Figure 4B and 4C. It was not uncommon for two block edges to share the same interior curve. In order for the mesh to merge successfully, both edges had to be projected to the same curve. Once all the block edges were projected to the curves, the final step was to project the exterior block faces to the model surface. The numbered surfaces imported from the IGES were grouped based on the partitioned section and defined under a new surface label using the sd command. This extra step was 
not strictly necessary, but it was a useful way of keeping the command line organized and legible. Exterior block surfaces were projected to the corresponding surface with the sfi command. Projecting block edges and surfaces defines the mesh's shape by adhering the block mesh to the model geometry. The mesh may not be a perfect representation of the geometry while it is in its unrefined state, but increasing the mesh density as in Figure 4D with the mseq command creates better congruency between the mesh and the geometry. Cartilage, meniscus, and ligaments were meshed with C3D8 linear block elements. The bones of the tibiofemoral model were modeled as discrete rigid shell elements to decrease computational time. 

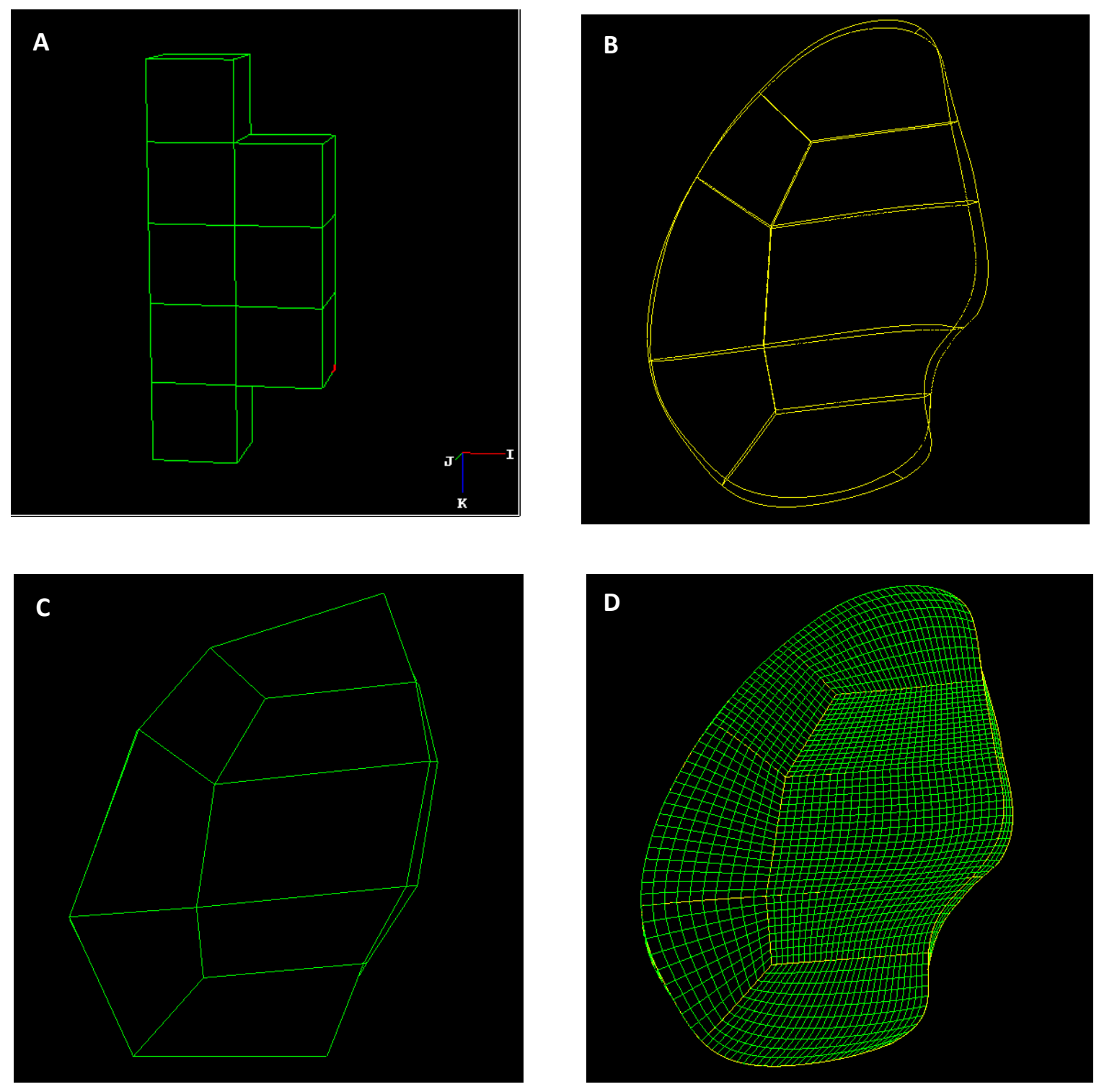

Figure 4: The computation block mesh $(\mathrm{A})$ is defined in Truegrid to match the partitioned sections created on the solid model. The partitions can be seen in (B) as curves defining the edges of the geometry. The computational blocks in (A) are projected to the curves and surfaces which produces a rough mesh in (C). The mesh is refined in (D) by increasing the mesh density. 


\subsection{FEA}

Abaqus is a finite element software suite that offers both pre- and postprocessing capabilities. Meshed parts were imported into Abaqus CAE and assembled as shown below in Figure 5. Material properties, interactions, and boundary conditions were defined in Abaqus CAE as well. Analysis was performed with Abaqus static/implicit solver with the nonlinear effects setting selected.

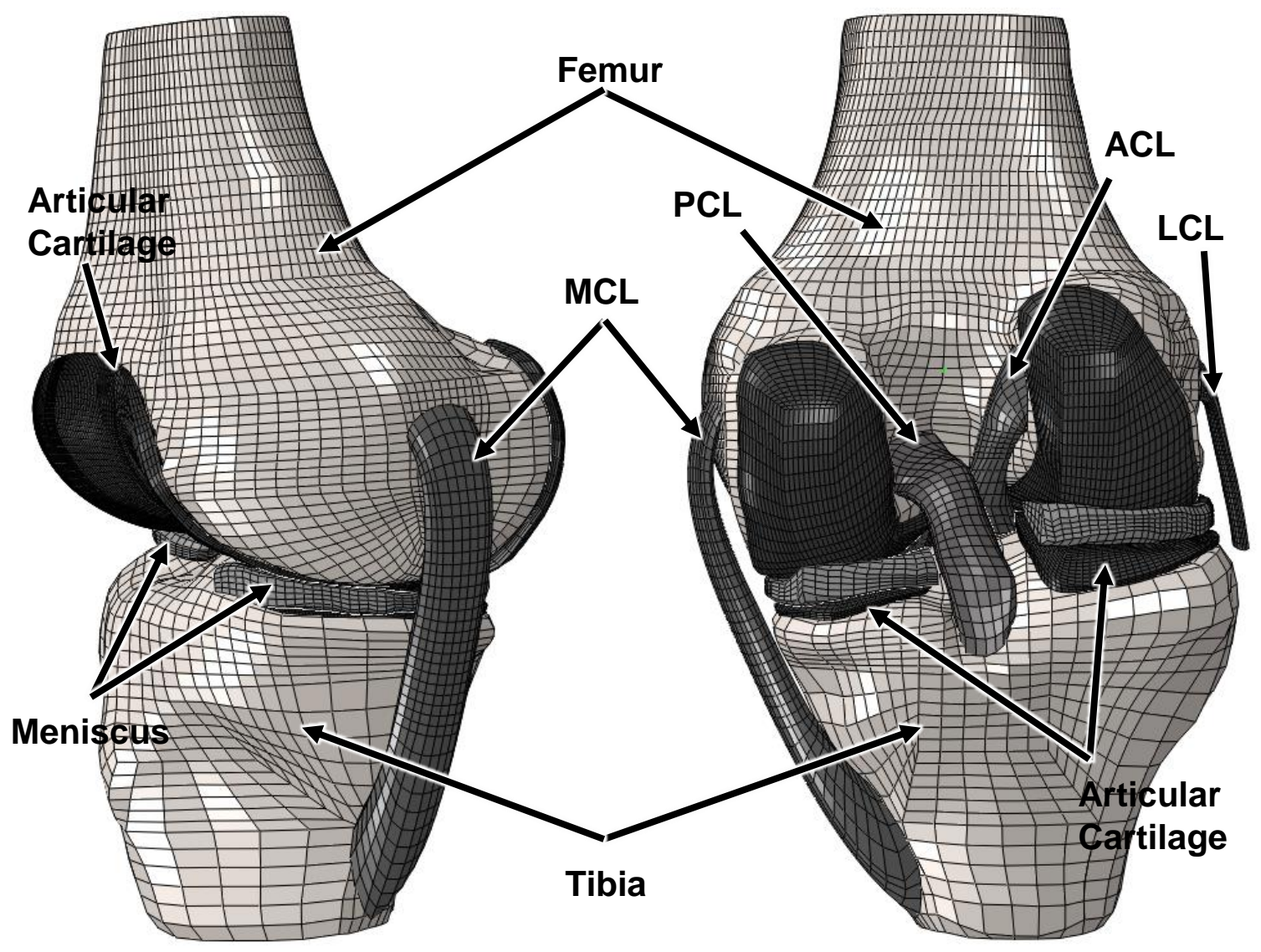

Figure 5: FE mesh of the tibiofemoral joint with anatomical structures labeled. The assembled FE model consists of the proximal tibia, distal femur, articular cartilage (femoral, and medial and lateral tibial), menisci (medial and lateral), and ligaments $(A C L=$ anterior cruciate ligament, $P C L=$ posterior cruciate ligament, $\mathrm{LCL}=$ lateral collateral ligament, $\mathrm{MCL}=$ medial collateral ligament). 


\subsubsection{Material Assignment}

The femur and tibia were modeled as rigid, non-deformable shells due to the greater stiffness of subchondral bone compared to soft tissue. This is a common approximation used in FE analysis and has been shown to negligibly affect results [21], [34].

The cruciate and collateral ligaments were assigned linear elastic, transverse isotropic material properties (Table 2). Ligaments are composed of parallel bundles of collagen fibers oriented to withstand tension in the direction of the ligament's length [35]. The high tensile strength of collagen fibers is the main contributor to the stiffness of the tissue, whereas proteoglycan ground substance provides minimal strength in the cross sectional plane of the ligament [35]. In order to create this specific directional dependence of the material properties, the local orientation of each ligament was specified in the Abaqus property module. The 1-direction was defined by manually selecting a longitudinal edge spanning the length of each individual structure, with the 2- and 3-directions forming the plane of isotropy.

The tibial and femoral articular cartilage were modeled as linear elastic, isotropic material with a Young's modulus $(\mathrm{E})$ of $15 \mathrm{MPa}$ and a Poisson's ratio $(v)$ of 0.475 (Table 2) [36]. In reality, articular cartilage is an extremely complex material that exhibits poro-viscoelastic properties [37]. Articular cartilage consists of a fluid-filled extracellular matrix, with interstitial fluid accounting for nearly $80 \%$ of its wet weight. The remaining $20 \%$ is a solid fiber matrix composed primarily of collagen and proteoglycans [38], [39]. Together, these constituents make up the 
main mechanical elements of articular cartilage [40]. Collagen fibers dispersed throughout articular cartilage exhibit depth dependent orientation and concentration, making articular cartilage both anisotropic and inhomogeneous [38-40]. Although complex material models better capture articular cartilage's time dependent behavior, previous studies have shown that the transient response of cartilage and meniscus can be accurately modeled using incompressible elastic properties and an equilibrium modulus [34], [41]. Activities such as walking, running, or going up stairs produce loading cycles lasting only fractions of a second. Considering that the transient response of cartilage lasts for hundreds to even thousands of seconds, a linear elastic material is sufficient for investigating the instantaneous load response for the purpose of this thesis [34] [42].

The lateral and medial meniscus were also assumed to be linear elastic, isotropic with a Young's modulus of $59 \mathrm{MPa}$ and a Poisson's ratio of 0.49 (Table 2). The meniscus exhibits viscoelastic material properties similar to articular cartilage, but with circumferentially oriented collagen fibers providing the main structure of the extracellular matrix [34]. Based on the rational described above, linear elastic material properties are a suitable approximation for investigating transient loading response [42]. 
Table 2: Element type, material type, and material properties assigned to each solid model geometry.

\begin{tabular}{|c|c|c|c|}
\hline Structure & Element Type & Material Type & $\begin{array}{c}\text { Material } \\
\text { Properties }\end{array}$ \\
\hline $\begin{array}{l}\text { Bone } \\
\text { Tibia, Femur }\end{array}$ & Shell & Rigid Body & -- \\
\hline $\begin{array}{l}\text { Cartilage } \\
\text { Femoral, Lateral } \\
\text { and Medial Tibial }\end{array}$ & $\begin{array}{l}\text { 3D hexahedral } \\
\text { C3D8 }\end{array}$ & $\begin{array}{l}\text { Linear Elastic } \\
\text { Isotropic }\end{array}$ & $\begin{array}{l}E=15 \mathrm{Mpa} \\
v=0.475\end{array}$ \\
\hline $\begin{array}{l}\text { Meniscus } \\
\text { Lateral and } \\
\text { Medial }\end{array}$ & $\begin{array}{l}\text { 3D hexahedral } \\
\text { C3D8 }\end{array}$ & $\begin{array}{l}\text { Linear Elastic } \\
\text { Isotropic }\end{array}$ & $\begin{array}{l}E=59 \mathrm{Mpa} \\
v=0.49\end{array}$ \\
\hline $\begin{array}{l}\text { Ligaments } \\
A C L, P C L, M C L \\
L C L\end{array}$ & $\begin{array}{l}\text { 3D hexahedral } \\
\text { C3D8 }\end{array}$ & $\begin{array}{l}\text { Linear Elastic } \\
\text { Transverse Isotropic }\end{array}$ & $\begin{array}{l}\mathrm{E}_{\mathrm{L}}=153.7 \mathrm{MPa} \\
\mathrm{E}_{\mathrm{T}}=5.1 \mathrm{Mpa} \\
v_{\mathrm{LT}}=1.4 \\
v_{\mathrm{T} T}=0.3\end{array}$ \\
\hline
\end{tabular}

\subsubsection{Interactions and Constraints}

Surface-to-surface contact interactions were defined between the following surface pairs: the femoral cartilage and each of the superior surfaces of the medial and lateral menisci; the lateral tibial cartilage and the distal surface of the lateral menisci; the medial tibial cartilage and the distal surface of the medial meniscus; and the outer surfaces of the ACL and PCL. Frictionless interaction with finite sliding was selected for each contact pair because articular cartilage is considered a low friction surface which primarily functions to provide smooth joint motion [43]. In addition, synovial fluid with the tibiofemoral compartment aids smooth joint motion by forming a fluid film that lubricates moving surfaces [44]. 
For these reasons, frictionless contact interactions within the tibiofemoral joint are a common and accepted assumption in computational modeling [43].

Tie constraints were used to anchor cartilage and ligaments to the bone. This type of constraint binds two surfaces together so that there is zero relative motion between them. For each contact pair, the surface region of the bone in contact with the soft tissue was selected and identified as the "master surface".

\subsubsection{Linear Springs}

Linear spring elements were used to replicate the fibrous tissue that attaches the meniscal horns to the tibia surface. The nodes on the medial and lateral faces of the meniscal horns were first assigned a kinematic coupling constraint to a single reference node at the center of each horn face. This particular type of constraint restricts the motion of the group of nodes to the rigid body motion of the single reference node. A spring element was then produced connecting the reference node of the horn to a node on the tibia plateau in line with the projected direction of the horn face as shown in Figure 6. Each meniscal spring was assigned a stiffness consistent with physiological studies [45]. Spring constants assigned to the meniscal horns are listed in Table 3.

Spring elements were also applied to the distal face of the LCL in order to simulate the stiffness of the inferior half of the LCL that was not included in this model. Springs were assigned to each node on the LCL distal face and stiffness constants were prescribed in the transverse and longitudinal directions [46], [47]. Due to the fact that spring constants sum in parallel, the total ligament stiffness 
was divided by the number of nodes to produce a spring stiffness per node.

Refer to Table 3 for LCL spring stiffness values.

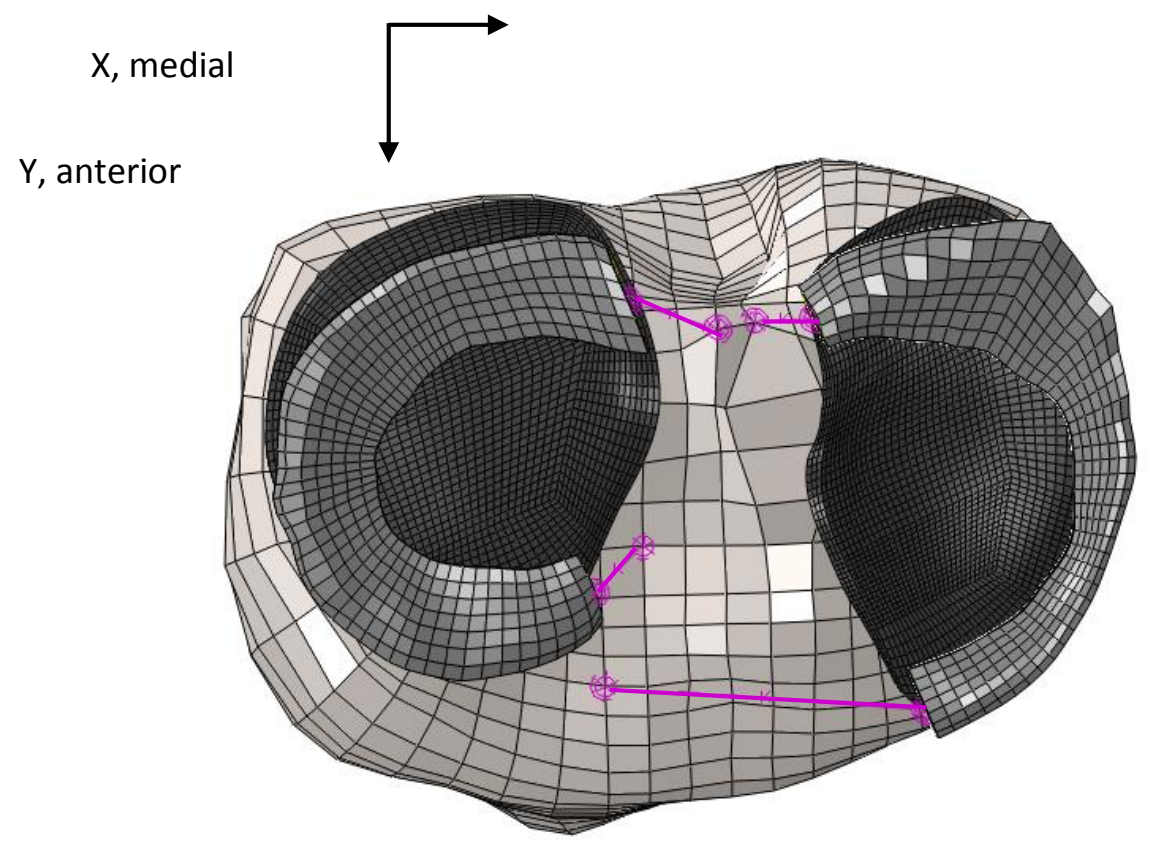

Figure 6: Spring elements of each meniscus horn are indicated in pink.

Table 3: Spring Stiffness constants assigned to the anterior and posterior horns of the menisci and the distal face of the LCL. The springs were either defined between two nodes in a line of action or in the Abaqus coordinate system.

\begin{tabular}{|c|c|c|}
\hline & $\begin{array}{c}\text { Stiffness } \\
(\mathbf{N} / \mathbf{m m})\end{array}$ & Direction \\
\hline $\begin{array}{c}\text { Medial Meniscus } \\
\text { Anterior Horn }\end{array}$ & 216 & $\begin{array}{c}\text { Line of Action } \\
\text { Line of Action }\end{array}$ \\
\hline $\begin{array}{c}\text { Posterior Horn } \\
\text { Lateral Meniscus } \\
\text { Anterior Horn } \\
\text { Posterior Horn }\end{array}$ & 128 & $\begin{array}{c}\text { Line of Action } \\
\text { Line of Action }\end{array}$ \\
\hline LCL & 207.2 & $\begin{array}{c}\text { Longitudinal } \\
\text { Transverse }\end{array}$ \\
\hline
\end{tabular}




\subsubsection{Loads and Moments}

Tibiofemoral contact forces and moments were obtained in collaboration with the Physical Activity Energetics/Mechanics Laboratory at Colorado State University, Fort Collins. Kinetic and kinematic data from obese and normal weight participants was processed using the methodology described by Haight et al. Briefly, each individual walked at a constant $1.25 \mathrm{~m} \mathrm{~s}^{-1}$ at a level incline on a dual-belt force measuring treadmill. Motion analysis software captured the participants' knee flexion angle and synced the motion with ground reaction forces recorded during gait. The data was then input into an OpenSim musculoskeletal model scaled to the mass and dimensions of each individual participant [19]. Joint Reaction analysis was performed in Opensim to calculate the resultant forces and moments at the tibiofemoral joint. Joint reaction calculations take into consideration the contribution of muscles and ligaments acting at the joint. Therefore, the resultant forces and moments used as inputs in this FE model are representative of the internal loads carried by the tibiofemoral joint and do not include the forces transferred through muscles or ligaments [48]. Joint reaction data from three obese and three normal weight participants were averaged for this study. The average obese weight was $936.3 \mathrm{~N}$ and the average normal weight was $606.3 \mathrm{~N}$. Full details regarding the weight and gender of the 6 individuals is provided below in Table 4 . The choice of three participants per weight group was supported using a power analysis calculation comparing the sagittal plane net muscle moments at the knee for obese and normal weight individuals [11]. Values were input into an online sample size calculator from 
DSS Research with a confidence level of $5 \%$ and a statistical power of $20 \%$ [49]. This calculation confirmed that the sample size was large enough to highlight differences between obese and normal weight gait.

Table 4: The gender and body weight of the obese and normal weight gait analysis participants.

\begin{tabular}{|c|c|}
\hline Gender & Body Weight (N) \\
\hline Normal Weight & 655.3 \\
F & 624.9 \\
M & 538.6 \\
F & \\
\hline Obese & 928.4 \\
F & 853.5 \\
F & 1027.1 \\
\hline
\end{tabular}

Six discrete points in the gait cycle were chosen for analysis based on the presence of high magnitude forces and moments. These points represent $0 \%$ stance (heel strike), 5\% stance, 25\% stance, 50\% stance, 75\% stance, and $100 \%$ stance (toe off). The joint contact forces, moments, and knee flexion angle corresponding to these phases of stance are listed in Tables 5 and 6 for normal weight and obese individuals, respectively.

Joint forces and moments were applied at a reference node located approximately at the midpoint between the femoral epicondyles. This point was selected to correspond to OpenSim's joint center about which the moments and forces were initially calculated [50]. OpenSim outputs joint loads in an anatomical reference plane; consequently, a local coordinate system was established in Abaqus that aligned with the anatomical position of the knee in order to simplify coordinate system transformations. The positive x-axis was defined in the medial 
direction, the positive $y$-axis was defined in the anterior direction, and the positive $z$-axis was defined to point distally in the direction of the longitudinal axis of the tibia.

Table 5: Summary of the flexion angles and loads for a normal weight individual at each of the 6 stages of stance analyzed in this study. Forces and moments are defined based on the local Abaqus coordinate system described above.

\begin{tabular}{|c|c|c|c|c|c|c|c|}
\hline \multirow[t]{2}{*}{$\begin{array}{c}\% \\
\text { Stance }\end{array}$} & \multirow{2}{*}{$\begin{array}{c}\text { Knee } \\
\text { Flexion } \\
\text { Angle } \\
\text { (degrees) }\end{array}$} & \multicolumn{3}{|c|}{$\begin{array}{l}\text { Joint Reaction Forces } \\
\text { (N) }\end{array}$} & \multicolumn{3}{|c|}{$\begin{array}{l}\text { Joint Reaction Moments } \\
\text { (N m })\end{array}$} \\
\hline & & $F_{X}$ & $F_{Y}$ & $F_{Z}$ & $\mathbf{M}_{\mathbf{X}}$ & $\mathbf{M}_{\mathbf{Y}}$ & $M_{z}$ \\
\hline 0 & 0.75 & 3.15 & -17.25 & 268.95 & 602.4 & 1108.2 & 952.6 \\
\hline 5 & 6.45 & 4.11 & -12.53 & 469.67 & 1442.7 & 3940.1 & 3801.7 \\
\hline 25 & 22.50 & 41.16 & -14.00 & 1391.84 & $\begin{array}{l}-717.3 \\
\end{array}$ & -13586.3 & 1655.1 \\
\hline 50 & 14.44 & 40.37 & 70.60 & 694.67 & 2441.0 & -8131.9 & 4633.5 \\
\hline 75 & 3.61 & 79.92 & 86.89 & 1882.19 & 7737.1 & -12911.9 & 10207.0 \\
\hline 100 & 49.74 & -11.85 & 19.53 & 234.25 & $\begin{array}{l}-177.8 \\
\end{array}$ & 4355.9 & -139.4 \\
\hline
\end{tabular}

Table 6: Summary of the flexion angles and loads for an obese individual at each of the 6 stages of stance analyzed in this study. Forces and moments are defined based on the local Abaqus coordinate system described above.

\begin{tabular}{|c|c|c|c|c|c|c|c|}
\hline $\begin{array}{c}\text { \% } \\
\text { Stance }\end{array}$ & $\begin{array}{c}\text { Knee } \\
\text { Flexion } \\
\text { Angle } \\
\text { (degrees) }\end{array}$ & \multicolumn{3}{|c|}{$\begin{array}{c}\text { Joint Reaction Forces } \\
\text { (N) }\end{array}$} & $F_{\mathbf{X}}$ & \multicolumn{3}{|c|}{$\mathbf{F}_{\mathbf{Y}}$} & \multicolumn{3}{c|}{$\begin{array}{c}\text { Joint Reaction Moments } \\
\text { (N mm) }\end{array}$} & $\mathbf{F}_{\mathbf{Z}}$ & $\mathbf{M}_{\mathbf{X}}$ & $\mathbf{M}_{\mathbf{Y}}$ & $\mathbf{M}_{\mathbf{Z}}$ \\
\hline 0 & 1.82 & 3.29 & -23.41 & 361.25 & 481.8 & -3482.5 & -859.9 \\
\hline 5 & 5.70 & 18.85 & -54.62 & 1140.29 & 3506.3 & 2624.2 & 5612.8 \\
\hline 25 & 18.43 & 65.85 & -3.36 & 1986.95 & -139.9 & -19266.1 & 3398.9 \\
\hline 50 & 7.06 & 61.64 & 90.61 & 956.73 & 3638.5 & -8489.7 & 6353.1 \\
\hline 75 & 4.84 & 93.09 & 179.17 & 2045.43 & 8477.4 & -8604.4 & 11651.0 \\
\hline 100 & 44.71 & -24.76 & 33.14 & 385.99 & -84.3 & 7440.5 & -1478.7 \\
\hline
\end{tabular}

\subsubsection{Boundary Conditions}

Boundary conditions were assigned to the tibia and femur during the alignment and loading stages of the FE analysis. The need for separate boundary conditions during alignment and loading arose due to excessive protruding of the menisci outside of the joint when assigning large flexion angles. 
In order to address this issue, the tibia was constrained in the 3 rotational degrees of freedom and free to translate anterior-posterior, medial-lateral, and in the longitudinal directions during alignment. Conversely, the femur was constrained by fixing translation in all 3 directions and by specifying a flexion angle for the rotational degree of freedom in the sagittal plane. Flexion angles of the femur at the investigated phases of stance are listed in Tables 5 and 6. In order to replicate the effects of a malalignment for the varus knee analysis, the femur was prescribed a rotation of $3^{\circ}$ medially in the frontal plane during alignment. Any remaining rotation degrees of freedom of the femur were unconstrained in the alignment stage.

During the loading stage, the tibia was completely constrained with zero degrees of freedom in its subsequent position following the alignment stage. It was necessary to keep the tibia fixed during loading because the joint reaction forces and moments were calculated in the tibial reference frame. Therefore, the kinetics and kinematics of the femur were defined in relation to the position of the tibia. The sagittal plane rotation of the femur remained fixed at the specified flexion angle defined in the alignment stage. All other degrees of freedom of the femur were unconstrained during loading for both the normal and varus malalignment analyses.

Finally, a boundary condition was defined on the distal face of the LCL to constrain the 3 rotational degrees of freedom. This allowed the LCL to translate based on the influence of the linear springs and was found to also drastically decrease computational time. 


\subsubsection{Mesh Convergence}

Mesh convergence studies were performed to determine the optimal mesh density of the femoral cartilage and the lateral and medial tibial cartilages. The menisci, ligaments, and bones were not included in the mesh convergence because the scope of this research is limited to stress in articular cartilage as it relates to $O A$.

Multiple versions of each articular cartilage mesh were produced by gradually increasing the mesh refinement in TrueGrid. The articular cartilage meshes were then analyzed consecutively in the tibiofemoral FE model. Finite element simulations for mesh convergence were accomplished by prescribing the knee flexion angle to $10.5^{\circ}$ and applying the forces and moments listed in Table 7, which are physiologically consistent for a normal weight individual at this particular phase of gait [51]. Boundary conditions and loads were applied as described in the previous sections.

Table 7: Summary of the loads implemented during the convergence study.

\begin{tabular}{|c|c|c|c|c|c|c|}
\hline \multirow{2}{*}{$\begin{array}{c}\text { Knee Flexion } \\
\text { Angle } \\
\text { (degrees) }\end{array}$} & \multicolumn{3}{|c|}{$\begin{array}{l}\text { Joint Reaction Forces } \\
\text { (N) }\end{array}$} & \multicolumn{3}{|c|}{$\begin{array}{l}\text { Joint Reaction Moments } \\
(\mathrm{N} \mathrm{mm})\end{array}$} \\
\hline & $F_{x}$ & $F_{Y}$ & $F_{z}$ & $\mathbf{M x}$ & MY & Mz \\
\hline 10.5 & -3.0 & 366.0 & 769.4 & -3001.0 & 1732.0 & -2593.0 \\
\hline
\end{tabular}

Contact pressure was evaluated at specific nodes on each of the articular cartilage surfaces. The locations of the nodes of interest are indicated in Figure 7. Selection of these nodes was based on their presence in high stress regions of the mesh as well as their fixed physical location on the articular cartilage surface. 

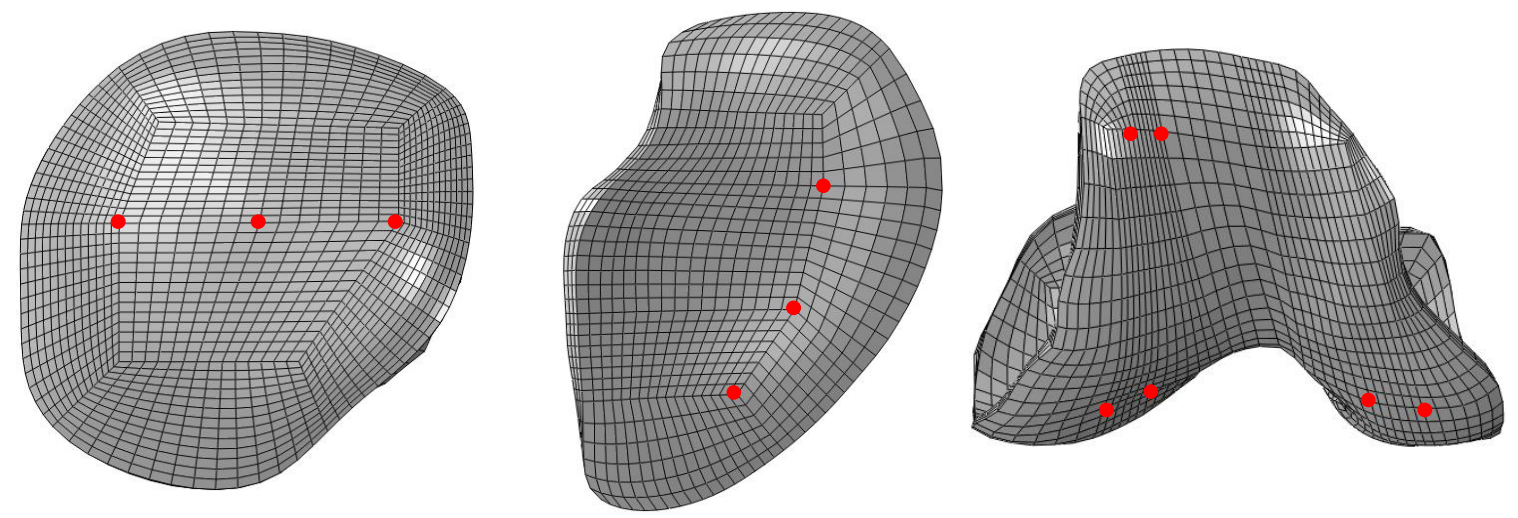

Figure 7: The location of the nodes analyzed in the mesh convergence study are indicated in red.

The results of the convergence study are shown below in Figure 8 as a plot of total mesh degrees of freedom versus contact pressure evaluated at a specific nodal location. Qualitatively, the graphs clearly indicate that the solutions approach a particular value as the mesh becomes more refined. Additional calculations were completed to confirm that the solution had in fact converged. Solution convergence was defined as a change of less than $1 \%$ between consecutively refined meshes for the lateral and medial tibial cartilages and less than $5 \%$ for the femoral cartilage meshes. Greater leniency was used to define convergence of the femoral cartilage mesh due to concerns about the high mesh density needed for the solution to converge within $1 \%$. Overall, the results indicate good convergences of the three cartilage meshes, which ensures the accuracy of the solutions output from the model. 

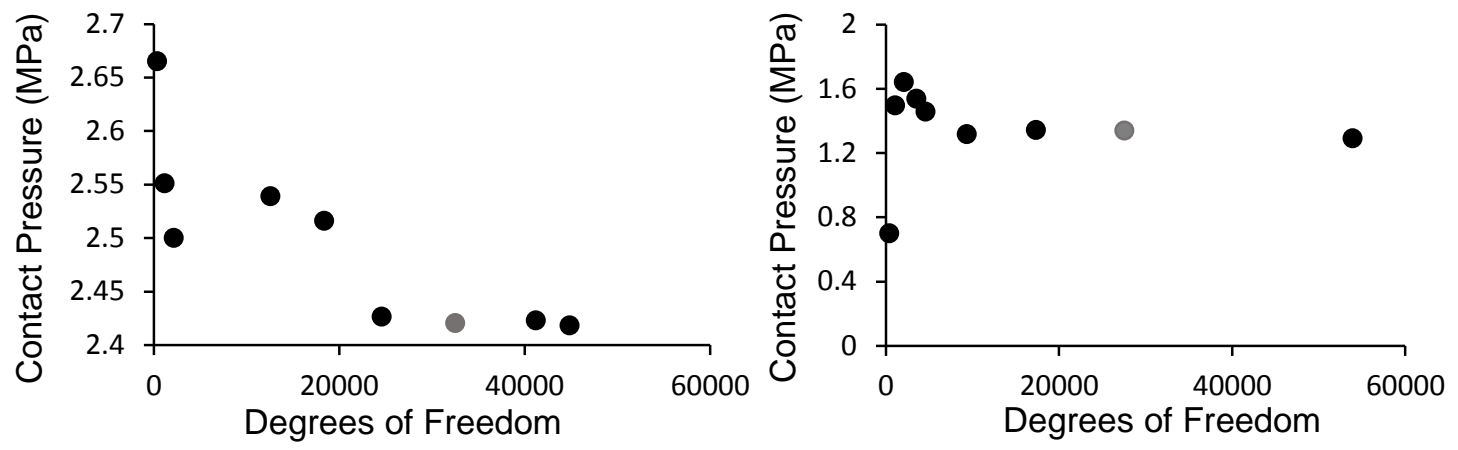

Femoral Cartilage, Proximal Node

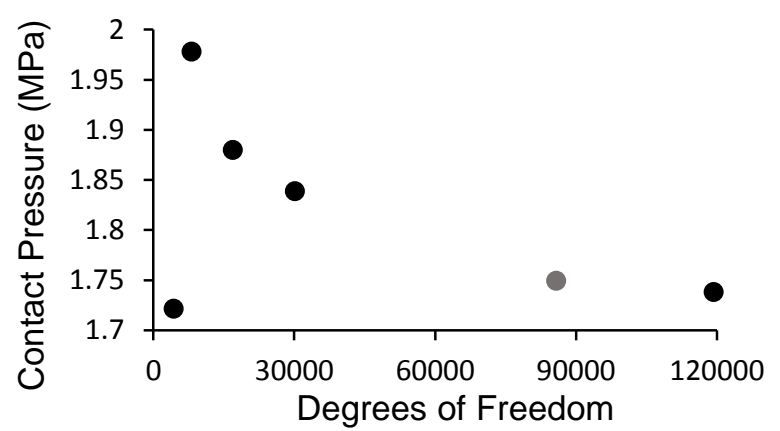

Figure 8: Convergence study results shown as a plot of contact pressure at the indicated convergence node vs the degrees of freedom of the mesh. Selected mesh degrees of freedom are indicated by the gray shaded markers.

The converged tibial cartilage meshes consist of 9,492 nodes with 28,476 degrees of freedom and 9,200 nodes with 27,600 degrees of freedom for the lateral and medial cartilages, respectively. The converged femoral cartilage mesh contains 28,595 nodes and 85,785 degrees of freedom. The final converged version of the tibiofemoral model consists of a total of 55,562 elements, 6,305 of which are attributed to the shell elements of the tibia and femur. The articular cartilage meshes account for a total of 35,970 elements and the menisci meshes total 2,772 elements. The remaining 10,785 elements are shared amongst the ligament meshes. 


\subsubsection{Damping Factor Stabilization}

Nonlinear static FE problems often have a high degree of instability. The extent of the nonlinearity in the system directly influences the number of iterations the FE solver requires to produce a converged solution [52]. Considering the high degree of nonlinear behavior in the current tibiofemoral model, coupled with its complex contact interactions, makes it of no surprise that initial simulation attempts terminated due to solution convergence errors.

One approach to stabilizing nonlinear static problems is by generating an artificial viscous force through manipulation of the damping factor. The damping factor is a numerical value that can be specified in Abaqus CAE within each step of the simulation. Abaqus calculates user applied damping much the same way the viscous force in a dashpot is calculated [52]. The damping factor is treated as a constant that is multiplied by the nodal velocity vector to produce a viscous force vector [52]. The viscous force is subtracted from the global equilibrium equations and therefore dissipates some of the energy from the model [52].

Manually increasing the damping factor to 0.002 addressed the solution convergence errors in the Abaqus simulations to great effect. However, damping factor stabilization can influence the accuracy of the final solution if the viscous factors become exceedingly large. As a precautionary measure, the ratio of the viscous force to total forces and the ratio of viscous damping energy to total strain energy in the model were calculated to confirm that the viscous terms were small compared to the model totals [52]. The results of these calculations are listed below in Tables 8 and 9. 
Table 8: The ratio of viscous force to total forces calculated for each subject type across all investigated phases of stance.

\begin{tabular}{|c|c|c|c|c|}
\hline $\begin{array}{c}\text { \% } \\
\text { Stance }\end{array}$ & Normal Weight & $\begin{array}{c}\text { Viscous Force : Total Force } \\
\text { Varus Weight , }\end{array}$ & Obese & $\begin{array}{c}\text { Obese, } \\
\text { Varus }\end{array}$ \\
\hline 0 & 0.005 & 0.014 & 0.005 & 0.015 \\
\hline 5 & 0.011 & 0.011 & 0.006 & 0.008 \\
\hline 25 & 0.005 & 0.006 & 0.004 & 0.004 \\
\hline 50 & 0.007 & 0.006 & 0.007 & 0.006 \\
\hline 75 & 0.007 & 0.006 & 0.009 & 0.008 \\
\hline 100 & 0.021 & 0.021 & 0.016 & 0.017 \\
\hline
\end{tabular}

Table 9: The ratio of viscous damping energy to total strain energy calculated for each subject type across all investigated phases of stance.

\begin{tabular}{|c|c|c|c|c|}
\hline & \multicolumn{4}{|c|}{ Viscous Damping Energy : Total Strain Energy } \\
\hline $\begin{array}{c}\text { \% } \\
\text { Stance }\end{array}$ & Normal Weight & $\begin{array}{c}\text { Normal Weight , } \\
\text { Varus }\end{array}$ & Obese & $\begin{array}{c}\text { Obese, } \\
\text { Varus }\end{array}$ \\
\hline 0 & $1.3 \mathrm{E}+02$ & $1.2 \mathrm{E}+03$ & $1.5 \mathrm{E}+02$ & $7.3 \mathrm{E}+02$ \\
\hline 5 & $4.8 \mathrm{E}+02$ & $7.4 \mathrm{E}+02$ & $2.4 \mathrm{E}+02$ & $3.6 \mathrm{E}+02$ \\
\hline 25 & $7.4 \mathrm{E}+02$ & $7.2 \mathrm{E}+02$ & $3.7 \mathrm{E}+02$ & $3.5 \mathrm{E}+02$ \\
\hline 50 & $7.6 \mathrm{E}+02$ & $7.4 \mathrm{E}+02$ & $3.0 \mathrm{E}+02$ & $3.2 \mathrm{E}+02$ \\
\hline 75 & $1.2 \mathrm{E}+02$ & $1.7 \mathrm{E}+02$ & $1.3 \mathrm{E}+02$ & $1.6 \mathrm{E}+02$ \\
\hline 100 & $4.6 \mathrm{E}+03$ & $4.6 \mathrm{E}+03$ & $3.8 \mathrm{E}+03$ & $3.8 \mathrm{E}+03$ \\
\hline
\end{tabular}

As can be seen in Table 8, the ratios of viscous force to total forces ranged from an order to magnitude of $10^{-3}$ to $10^{-2}$. These results suggest that the viscous forces in the model are sufficiently small enough that they do not dominate in the model. However, the ratios of viscous damping energy to total strain energy in Table 9 are of significant concern. In general, it is recommended that the viscous energy ratios not exceed $10^{-4}$ in order to ensure that the model is producing accurate solutions [52]. Viscous damping energy can become magnified if the model experiences a large amount of motion, as is the case with 
the tibiofemoral model in this study [52]. Although the ratios in Table 8 and 9 have conflicting implications, it is very likely that the tibiofemoral model solutions are inaccurate due to the manually specified damping factor [52]. The consequences of this finding are discussed in greater detail in Section 4.2.1.

\subsubsection{FE Model Validation}

The tibiofemoral FE model was validated against experimental cadaver studies found in a review of the literature. Studies were only included in the validation analysis if they met the following criteria: First, they must report contact pressure and area measured at the articular cartilage surface in the tibiofemoral compartment. Second, the cadaver knees could not exhibit any visual signs of cartilage deterioration. Third, the ligaments, menisci, and articular cartilage of the tibiofemoral joint must have been left intact during the experiment. Finally, the cadaver knees must have been loaded and constrained in such a way that could be replicated in the boundary and loading conditions of the FE model.

Validation analyses were performed by applying either a $500 \mathrm{~N}, 1000 \mathrm{~N}$, or $1800 \mathrm{~N}$ load to the tibiofemoral FE model while also prescribing a knee flexion angle of $0^{\circ}, 15^{\circ}$, or $30^{\circ}$ for a total of eight distinct configurations. The tibia and femur boundary conditions were dictated by the test protocol used in the experimental studies. Loads were applied to the reference node between the femoral condyles, unless an alternate loading apparatus was specified in the study. 


\subsubsection{Output Variables}

The output variables for this FE model include contact pressure (CPRESS) and contact area (CAREA). These parameters were used to analyze the risk of cartilage damage and OA initiation. As stated in the previous section, viscous force (VF), total forces (TF), viscous damping energy (ALLSD), and internal energy (ALLIE) were also included as output variables. These parameters were used to determine the effect of the damping factor on the accuracy of the solution. 


\section{CHAPTER 3}

\section{RESULTS}

\subsection{Validation Results}

The validation results presented in this section were produced using the methodology described in Section 2.3.8. All analyses were performed in the Abaqus static/implicit solver with the nonlinear effects setting selected and with the material properties, constraints, interactions, and linear springs described in Methods.

\subsubsection{Maximum Contact Pressure Validation}

Contact pressure was evaluated at the surface nodes of the medial and lateral articular cartilage in the tibiofemoral FE model. In order to more accurately replicate the experimental results, the FE model nodal contact pressure was evaluated over an area of either $4 \mathrm{~mm}^{2}$ or $1.6 \mathrm{~mm}^{2}$, (depending on the specific experimental sensor resolution) and then averaged to produce the maximum contact pressure values in Table 10. Experimental maximum contact pressure was evaluated in the medial and lateral tibial compartments of cadaver knees using either pressure sensors or pressure sensitive film.

There was very favorable correlation between the FE and experimental maximum contact pressures at $0^{\circ}$ of knee flexion for all loading conditions. Both medial and lateral cartilage FE results were within one standard deviation of the values reported in Seitz et al. [53] and Marzo et al. [54]. At $0^{\circ}$ knee flexion and $500 \mathrm{~N}$, the medial and lateral FE maximum contact pressures differed from the experimental mean reported by Seitz by only $9.1 \%$ and $17.3 \%$, respectively. The 
percent difference between the medial and lateral FE contact pressures and Seitz's experimental mean increased to $14.2 \%$ and $25.3 \%$, respectively, at $0^{\circ}$ flexion and $1000 \mathrm{~N}$, which may imply that the FE model has greater accuracy with smaller loads at low flexion angles. However, both the medial and lateral FE contact pressures differed by less than $6 \%$ of the experimental mean reported by Marzo when loading was increased to 1800 N. Marzo and Seitz applied different boundary conditions during loading and used sensors with different resolutions, so it is difficult to make a direct comparison between the validation results from the two studies.

The FE results did not validate as well against Morimoto et al.'s [55] experimental results when the test fixture boundary conditions were strictly replicated in the FE model. These initial FE model validation attempts produced medial and lateral contact pressures that were within two and three standard deviations of Morimoto's experimental mean, respectively. However, it was discovered through trial and error that releasing the abduction/adduction rotational constraint on the femur model produced results that more closely matched the experimental contact pressures reported by Morimoto. As can be seen in Table 10, including this extra degree of freedom decreased the medial contact pressure to within one standard deviation of the experimental mean and increased the lateral contact pressure to within two standard deviations of the experimental mean. The effect of the abduction/adduction rotational constraint in the Morimoto validation analysis can also be observed in the FE model results at $15^{\circ}$ and $30^{\circ}$ flexion. In both cases, the FE medial and lateral contact pressures 
results were closer to the experimental mean when abduction/adduction of the femur was unconstrained. The observations made during the Morimoto validation analysis highlight a potential weakness in the FE model in that the results output from the model are influenced by slight changes in boundary conditions.

At $30^{\circ}$ flexion, the FE medial contact pressure was within two standard deviations of Seitz's experimental mean at $500 \mathrm{~N}$ loading and within one standard deviation at $1000 \mathrm{~N}$ loading. Similarly, the FE medial contact pressure very closely matched the Morimoto results at $30^{\circ}$ flexion. The FE lateral contact pressure did not validate against Seitz's results at $30^{\circ}$ flexion for either the $500 \mathrm{~N}$ or $1000 \mathrm{~N}$ loading cases. However, the FE lateral contact pressure was within two standard deviations of the experimental mean reported by Morimoto at $30^{\circ}$ flexion and $1000 \mathrm{~N}$ loading.

The results of this validation analysis suggest that the FE model can most accurately predict medial and lateral maximum contact pressures at low knee flexion angles. At $0^{\circ}$ flexion, the FE model's medial contact pressure results successfully validated to within two standard deviations for all four experimental results. The FE model's lateral contact pressure results were nearly as successful, validating to within two standard deviations for three out of four of the experimental studies. The FE medial and lateral contact pressure both validated to within two standard deviations at $15^{\circ}$ flexion; however, only one experimental study reporting contact pressure at $15^{\circ}$ flexion was found in the literature so there is less support backing the FE model results. Of the six phases of stance analyzed in this thesis, four have knee flexion angles of $15^{\circ}$ or less for both 
normal weight and obese gait. Knee flexion angles become larger towards the later phase of stance (toe-off), at which point the FE model's contact pressure results may become less accurate based on the $30^{\circ}$ flexion validation analysis.

Table 10: A summary of the maximum contact pressure validation analysis results. The maximum contact pressure determined from the FE model is compared to the maximum contact pressure values obtained from experimental cadaver studies.

\begin{tabular}{|c|c|c|c|c|c|}
\cline { 5 - 6 } \multicolumn{2}{c|}{} & \multicolumn{2}{c|}{ Medial Tibial Cartilage } & \multicolumn{2}{c|}{ Lateral Tibial Cartilage } \\
\hline Study & $\begin{array}{c}\text { Load } \\
\text { (N) }\end{array}$ & $\begin{array}{c}\text { Max Contact } \\
\text { Pressure } \\
(\mathrm{Mpa})\end{array}$ & $\begin{array}{c}\text { FE Max } \\
\text { Contact } \\
\text { Pressure } \\
(\mathrm{Mpa})\end{array}$ & $\begin{array}{c}\text { Experimental } \\
\text { Max Contact } \\
\text { Pressure } \\
(\mathrm{Mpa})\end{array}$ & $\begin{array}{c}\text { FE Max } \\
\text { Contact } \\
\text { Pressure } \\
(\mathrm{Mpa})\end{array}$ \\
\hline Seitz [53] & 500 & $1.78 \pm 0.60$ & 1.95 & $1.79 \pm 0.82$ & 2.13 \\
\hline Seitz [53] & 1000 & $2.82 \pm 0.87$ & 3.25 & $3.04 \pm 1.32$ & 3.92 \\
\hline Morimoto [55] & 1000 & $4.88 \pm 1.20$ & 6.34 & $5.66 \pm 1.20$ & 2.34 \\
\hline $\begin{array}{c}\text { Morimoto [55] } \\
\text { (abd/add } \\
\text { rotation) }\end{array}$ & 1000 & $4.88 \pm 1.20$ & 4.08 & $5.66 \pm 1.20$ & 3.35 \\
\hline Marzo & 1800 & $3.841 \pm 1.24$ & 3.95 & $5.081 \pm 0.769$ & 4.82 \\
\hline
\end{tabular}

$15^{\circ}$ Knee Flexion

\begin{tabular}{|c|c|c|c|c|c|}
\hline $\begin{array}{c}\text { Morimoto [55] } \\
\begin{array}{c}\text { Morimoto [55] } \\
\text { (abd/add } \\
\text { rotation) }\end{array}\end{array}$ & 1000 & $4.77 \pm 0.75$ & 3.81 & $4.86 \pm 1.15$ & 6.82 \\
\hline
\end{tabular}

\begin{tabular}{|c|c|c|c|c|c|}
\hline \multicolumn{2}{|c|}{$\mathbf{0}^{\circ}$ Knee Flexion } & & & \\
\hline Seitz [53] & 500 & $1.92 \pm 0.68$ & 0.88 & $1.50 \pm 0.72$ & 4.68 \\
\hline Seitz [53] & 1000 & $3.21 \pm 1.20$ & 2.09 & $2.64 \pm 1.29$ & 6.90 \\
\hline Morimoto [55] & 1000 & $4.39 \pm 0.97$ & 3.58 & $5.07 \pm 1.15$ & 6.82 \\
\hline $\begin{array}{c}\text { Morimoto [55] } \\
\text { (abd/add } \\
\text { rotation) }\end{array}$ & 1000 & $4.39 \pm 0.97$ & 4.77 & $5.07 \pm 1.15$ & 5.02 \\
\hline
\end{tabular}

\subsubsection{Contact Area Validation}

FE model contact area was evaluated at the surface of the medial and lateral tibial articular cartilage models. The FE model values in Table 11 are a 
reflection of the amount of contact between the tibial articular cartilage, meniscus, and femoral articular cartilage models. The experimental contact area was obtained from the same published sources as in Section 3.1.1. Pressure sensors or pressure sensitive film were used in the experiments to obtain the contact area in the medial and lateral tibial compartments.

The validation results in Table 11 show inconsistent correlation between FE contact area results and the contact area determined in experimental studies. At $0^{\circ}$ knee flexion, the medial and lateral FE contact areas were within one standard deviation of the mean reported by Seitz at $500 \mathrm{~N}$ loading. When the load was increased to $1000 \mathrm{~N}$, the medial FE contact area deviated by two standard deviations from the Seitz's mean contact area. In comparison, the lateral and medial FE contact areas were within two and three standard deviations, respectively, of the average contact area reported by Morimoto for the same loading and flexion angle conditions. Releasing the abduction/adduction rotational constraint in the Morimoto analysis decreased the percent difference between the medial FE model and experimental value by $13.8 \%$, bringing the medial FE contact area to within two standard deviations of Moroimoto's reported contact area. Similarly, the percent difference between the lateral FE contact area and the Morimoto's reported contact area decreased by $16.6 \%$ when the abduction/adduction rotational constraint was released. Neither the medial nor the lateral FE contact pressures validated against the results reported by Marzo for $0^{\circ}$ flexion and $1000 \mathrm{~N}$ load. 
When the knee flexion angle was increased to $15^{\circ}$ and loading maintained at $1000 \mathrm{~N}$, the lateral FE contact area was within two standard deviations of the experimental contact area. In comparison, the medial FE contact area's deviation from the experimental mean was greater. Contrary to previous observations, releasing the abduction/adduction rotational constraint in the FE model validation analysis did not bring the FE model contact area closer to the experimental mean values.

The lateral FE contact area correlated well with the Seitz contact areas at $30^{\circ}$ flexion. For both the $500 \mathrm{~N}$ and $1000 \mathrm{~N}$ cases, the lateral FE model results were within one standard deviation of the Seitz mean contact area. In comparison, the lateral FE model results validated to within two standard deviations of the Morimoto experimental results at the same flexion and $1000 \mathrm{~N}$ load. The medial FE contact area did not validate to the experimental contact areas reported by Seitz at $30^{\circ}$ flexion for either loading cases. Additionally, the medial FE contact area only came within three standard deviations of the Morimoto experimental mean.

Overall, the lateral FE contact area validated more successfully to the values reported in the literature than the medial FE contact area. The results discussed in this section demonstrate the limitations of the tibiofemoral FE model at predicting contact pressure at the articular cartilage surface. 
Table 11: A summary of the contact area validation analysis results. The contact area determined from the FE model is compared to the contact area values obtained from experimental cadaver studies.

\begin{tabular}{|c|c|c|c|c|c|}
\hline & & \multicolumn{2}{|c|}{ Medial Tibial Cartilage } & \multicolumn{2}{|c|}{ Lateral Tibial Cartilage } \\
\hline \multicolumn{2}{|c|}{$0^{\circ}$ Knee Flexion } & \multirow{2}{*}{$\begin{array}{l}\text { Experimental } \\
\text { Contact Area } \\
\qquad\left(\mathrm{mm}^{2}\right)\end{array}$} & \multirow{2}{*}{$\begin{array}{c}\text { FE Model } \\
\text { Contact } \\
\text { Area } \\
\left(\mathrm{mm}^{2}\right)\end{array}$} & \multirow{2}{*}{$\begin{array}{l}\text { Experimental } \\
\text { Contact Area } \\
\qquad\left(\mathrm{mm}^{2}\right)\end{array}$} & \multirow{2}{*}{$\begin{array}{c}\text { FE Model } \\
\text { Contact } \\
\text { Area } \\
\left(\mathrm{mm}^{2}\right)\end{array}$} \\
\hline Study & $\begin{array}{l}\text { Load } \\
(\mathrm{N})\end{array}$ & & & & \\
\hline Seitz [53] & 500 & $319.08 \pm 122.99$ & 213.16 & $327.69 \pm 163.17$ & 340.77 \\
\hline Seitz [53] & 1000 & $387.33 \pm 106.59$ & 266.66 & $373.19 \pm 157.90$ & 394.43 \\
\hline Morimoto [55] & 1000 & $578.31 \pm 177.09$ & 218.02 & $443.05 \pm 120.60$ & 254.62 \\
\hline $\begin{array}{l}\text { Morimoto [55] } \\
\text { (abd/add } \\
\text { rotation) }\end{array}$ & 1000 & $578.31 \pm 177.09$ & 257.85 & $443.05 \pm 120.60$ & 303.60 \\
\hline Marzo [54] & 1800 & $594.0 \pm 59.0$ & 384.58 & $571.0 \pm 80.0$ & 526.67 \\
\hline
\end{tabular}

\begin{tabular}{|c|c|c|c|c|c|}
\hline \multicolumn{6}{|c|}{$15^{\circ}$ Knee Flexion } \\
\hline Morimoto [55] & 1000 & $488.47 \pm 140.34$ & 168.55 & $495.3 \pm 146.49$ & 248.55 \\
\hline $\begin{array}{c}\text { Morimoto [55] } \\
\text { (abd/add } \\
\text { rotation) }\end{array}$ & 1000 & $488.47 \pm 140.34$ & 147.90 & $495.3 \pm 146.49$ & 218.74 \\
\hline
\end{tabular}

\begin{tabular}{|c|c|c|c|c|c|}
\hline \multicolumn{2}{|c|}{$30^{\circ}$ Knee Flexion } \\
\hline Seitz [53] & 500 & $344.26 \pm 126.23$ & 23.19 & $302.46 \pm 127.05$ & 179.83 \\
\hline Seitz [53] & 1000 & $407.58 \pm 89.39$ & 70.40 & $363.64 \pm 145.45$ & 236.12 \\
\hline Morimoto [55] & 1000 & $449.91 \pm 156.76$ & 116.77 & $507.05 \pm 189.64$ & 197.7 \\
\hline $\begin{array}{c}\text { Morimoto [55] } \\
\text { (abd/add } \\
\text { rotation) }\end{array}$ & 1000 & $449.91 \pm 156.76$ & 143.25 & $507.05 \pm 189.64$ & 188.28 \\
\hline
\end{tabular}

3.2 Gait Analysis Results

The maximum contact pressure recorded at the surface of the medial and lateral tibial cartilage for each subject type across all phases of stance are displayed in Figures 9 and 10. As can be seen in Figure 9, contact pressure on the medial cartilage was at a minimum for normal weight (NW) loading conditions and at a maximum for obese loading conditions with varus alignment $(\mathrm{OB}, \mathrm{V})$ between $0 \%$ and $50 \%$ stance. Between heel strike and mid-stance, normal 
weight loading conditions with varus malalignment $(\mathrm{NW}, \mathrm{V})$ produced greater medial contact pressure than NW conditions; likewise, OB,V conditions produced greater medial contact pressure than obese (OB) loading conditions. The percent change between NW and NW,V medial contact pressure was fairly consistent at approximately $6-8 \%$ from heel strike to $25 \%$ stance. At $50 \%$ stance, the difference in medial contact pressure between NW and NW,V increased slightly, producing a change of $12.7 \%$. The greatest difference in medial contact pressure between $\mathrm{OB}$ and $\mathrm{OB}, \mathrm{V}$ loading conditions occurred at $25 \%$ stance with a difference of $11.1 \%$.

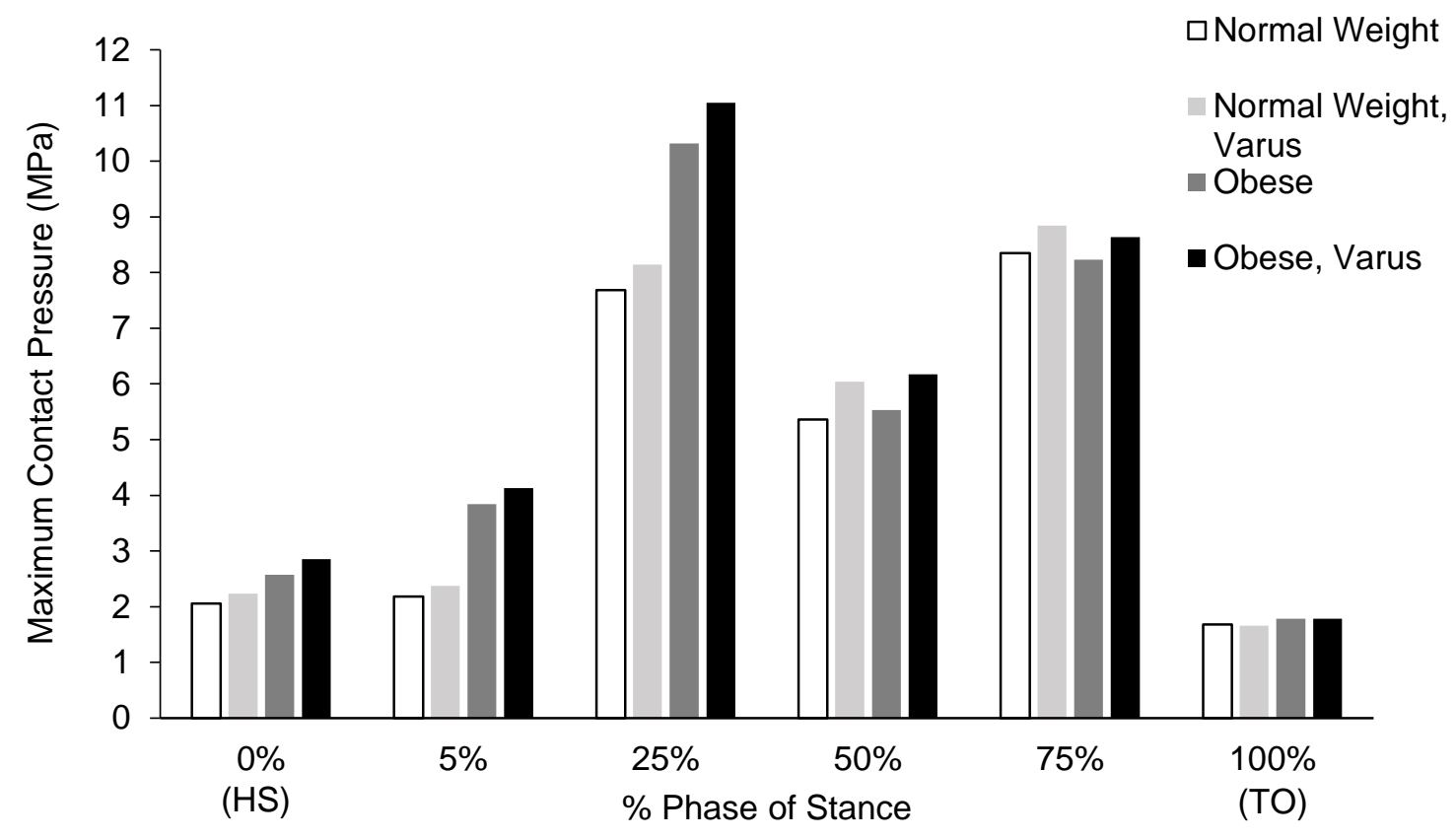

Figure 9: The bar graph of maximum contact pressure on the surface of the medial tibial cartilage as predicted by the tibiofemoral FE model. Maximum contact pressure is displayed for each subject type across all investigated phases of stance. $\mathrm{HS}=$ heel strike and TO = toe off. 
Past $50 \%$ stance, the medial contact pressure results deviate slightly from the observed trends described above. At $75 \%$ stance, medical contact pressure was greatest for NW,V and least for OB. The difference between NW and OB medial contact pressure was also at a minimum of $1.4 \%$ during $75 \%$ stance. Interestingly, medial contact pressure for $\mathrm{OB}$ and $\mathrm{OB}, \mathrm{V}$ were identical in value at $100 \%$ stance. Also, the medial contact pressure for NW was greater than NW,V at $100 \%$ stance, which was not observed at any other phase of stance.

The maximum contact pressure recorded on the surface of the lateral tibial cartilage is displayed in Figure 10. From heel strike to $5 \%$ stance, both the NW and $\mathrm{OB}$ lateral contact pressures were greater than the $\mathrm{NW}, \mathrm{V}$ and $\mathrm{OB}, \mathrm{V}$ lateral contact pressures, respectively. Lateral contact pressure at heel strike was greatest overall for NW, but OB lateral contact pressure surpassed all other conditions at $5 \%$. There was a $55 \%$ relative change between NW and OB lateral contact pressure at $5 \%$ stance, which is by far greater than at any other phase of stance.

During the middle phases of stance $(25 \%-75 \%)$ both NW,V and OB,V lateral contact pressures exceeded their normal aligned counterparts. Additionally, varus malalignment produced approximately equivalent relative increases in contact pressure for NW and OB in each modeled phase of stance between $25 \%$ and $75 \%$. The lateral contact pressures from $25 \%$ to $75 \%$ stance was consistently greatest for $\mathrm{OB}, \mathrm{V}$ and least for NW.

At toe-off, lateral contact pressure was similar between the normal and varus malaligned conditions for both weight groups. The greatest lateral contact 
pressure was observed for $\mathrm{OB}$, but the relative difference between $\mathrm{OB}$ and $\mathrm{OB}, \mathrm{V}$ was only $2.6 \%$. Similarly, NW had the smallest magnitude of lateral contact pressure at toe-off, but the difference between NW and NW,V was only $0.4 \%$.

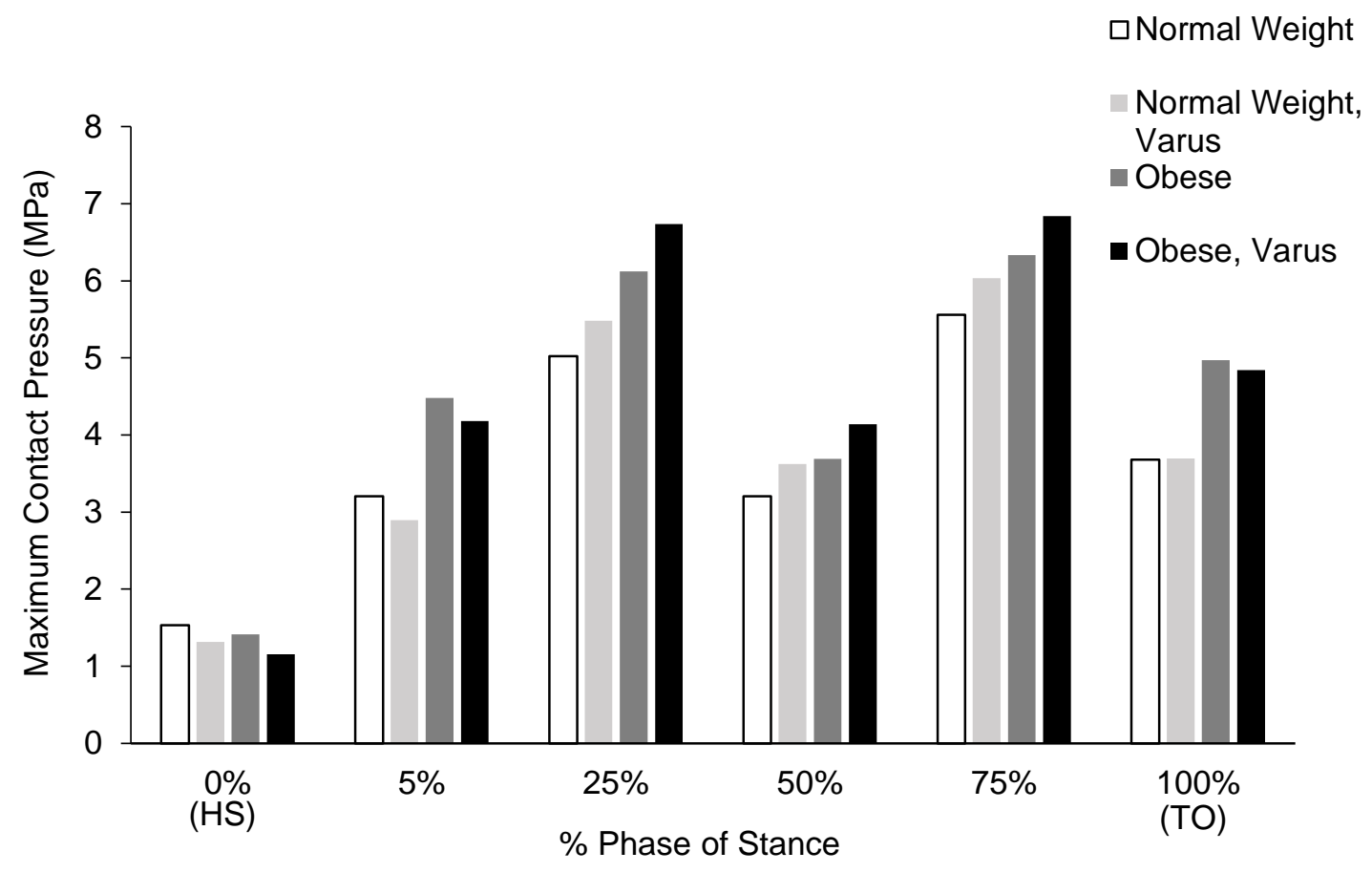

Figure 10: The bar graph of maximum contact pressure on the surface of the lateral tibial cartilage as predicted by the tibiofemoral FE model. Maximum contact pressure is displayed for each subject type across all investigated phases of stance. $\mathrm{HS}=$ heel strike and TO = toe off.

Many of the trends observed in the medial and lateral contact pressure data can also be seen in the femoral cartilage contact pressure results in Table 12. For example, varus malalignment produced greater maximum femoral contact pressure values than neutral alignment for both weight conditions at the majority of the phases of stance. The only exception to this trend occurred at $5 \%$ stance, in which case both NW and OB had greater femoral contact pressure than $\mathrm{NW}, \mathrm{V}$ and $\mathrm{OB}, \mathrm{V}$, respectively. The greatest femoral contact pressure was 
observed at $25 \%$ stance for $\mathrm{OB}$ and $\mathrm{OB}, \mathrm{V}$ and $75 \%$ stance for $\mathrm{NW}$ and $\mathrm{NW}, \mathrm{V}$. Medial and lateral tibial contact pressure was also greatest at $75 \%$ stance for NW and $\mathrm{NW}, \mathrm{V}$; however, $\mathrm{OB}$ and $\mathrm{OB}, \mathrm{V}$ had the greatest medial contact pressure at $25 \%$ and the greatest lateral contact pressure at $75 \%$ stance. Femoral contact pressure was most similar between neutral alignment and varus alignment at toeoff for both weight conditions, which is consistent with the observations made from the medial and lateral contact pressure data.

Table 12: The maximum contact pressure on the surface of the femoral cartilage as predicted by the tibiofemoral FE model. The location of the maximum contact pressure is indicated as either the medial or lateral femoral condyle. CPress $=$ contact pressure, $\mathrm{HS}=$ heel strike, and TO = toe off.

\begin{tabular}{|c|c|c|c|c|c|c|c|c|}
\cline { 2 - 9 } \multicolumn{1}{c|}{} & \multicolumn{2}{c|}{ Normal Weight } & \multicolumn{2}{c|}{$\begin{array}{c}\text { Normal Weight, } \\
\text { Varus }\end{array}$} & \multicolumn{2}{c|}{ Obese } & \multicolumn{2}{c|}{ Obese, Varus } \\
\hline $\begin{array}{c}\% \\
\text { Stance }\end{array}$ & $\begin{array}{c}\text { Max } \\
\text { CPress } \\
\text { (Mpa) }\end{array}$ & Condyle & $\begin{array}{c}\text { Max } \\
\text { CPress } \\
(\mathrm{Mpa})\end{array}$ & Condyle & $\begin{array}{c}\text { Max } \\
\text { CPress } \\
(\mathrm{Mpa})\end{array}$ & Condyle & $\begin{array}{c}\text { Max } \\
\text { CPress } \\
(\mathrm{Mpa})\end{array}$ & Condyle \\
\hline $\begin{array}{c}0 \% \\
(\mathrm{HS})\end{array}$ & 1.547 & Medial & 1.927 & Medial & 2.433 & Medial & 2.772 & Medial \\
\hline $5 \%$ & 3.026 & Lateral & 2.707 & Lateral & 4.309 & Lateral & 3.944 & Lateral \\
\hline $25 \%$ & 7.33 & Medial & 7.733 & Medial & 10 & Medial & 10.62 & Medial \\
\hline $50 \%$ & 5.172 & Medial & 5.78 & Medial & 5.3 & Medial & 5.976 & Medial \\
\hline $75 \%$ & 7.741 & Medial & 8.307 & Medial & 7.715 & Medial & 8.166 & Medial \\
\hline $\begin{array}{c}100 \% \\
(\mathrm{TO})\end{array}$ & 3.447 & Lateral & 3.458 & Lateral & 4.559 & Lateral & 4.509 & Lateral \\
\hline
\end{tabular}

As Table 12 shows, the maximum contact pressure location on the femoral condyles did not vary by weight group or alignment. Maximum femoral contact pressure was consistently located on the medial femoral condyle for $0 \%$, $25 \%, 50 \%$ and $75 \%$ stance and on the lateral femoral condyle for $5 \%$ and $100 \%$ of stance. 
Contour plots of contact pressure for each subject type across all phases of stance are provided in Appendix A. The images show that variations in load due to obesity did not cause drastic changes in the location of high pressure regions on the medial and lateral tibia cartilage surface in comparison to the normal weight control. However, obese loading did broaden the region of articular cartilage exposed to contact pressure. In Figure 11, the effect of obesity is visible on the lateral, medial, and femoral cartilage contour plots when comparing obese and normal weight subjects. Contact pressure is not only greater for the obese subject, but the region of exposed cartilage extends farther across the cartilage surface. Along these same lines, varus malalignment did not significantly alter the location or area of the high pressure region, but it did redistribute contact pressure from the lateral cartilage to the medial cartilage at $25 \%$ stance for both normal weight and obese loading conditions. 
Figure 11: Contact pressure in MPa on the superior surface of the lateral and medial tibial cartilage and the inferior aspect of the femoral cartilage for each subject type at $25 \%$ stance. $(A=$ anterior and $\mathrm{P}=$ posterior direction).

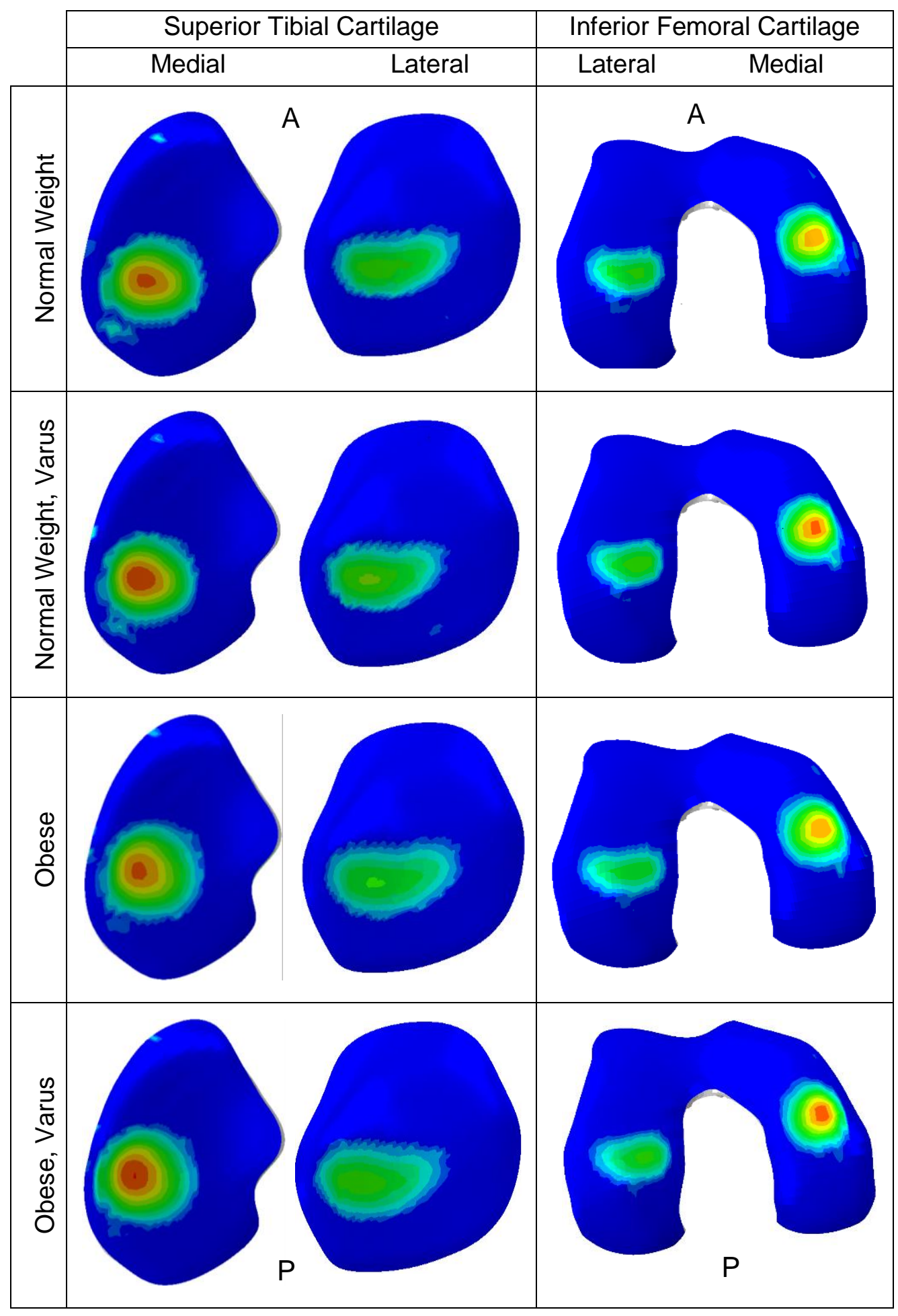


Table 13: Contact areas at the surface of the medial, lateral, and femoral articular cartilage for each subject type across all investigated phases of stance. HS = heel strike and $\mathrm{TO}=$ toe off.

\begin{tabular}{|c|c|c|c|c|c|c|c|}
\hline & $\%$ stance: & $\begin{array}{c}0 \% \\
(H S)\end{array}$ & $5 \%$ & $25 \%$ & $50 \%$ & $75 \%$ & $\begin{array}{c}100 \% \\
\text { (TO) }\end{array}$ \\
\hline \multirow{3}{*}{ 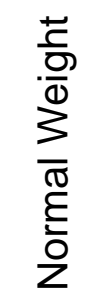 } & medial & 147.1 & 106.1 & 197.0 & 150.1 & 280.5 & 55.6 \\
\hline & lateral & 236.9 & 240.4 & 179.8 & 117.8 & 298.6 & 96.3 \\
\hline & femoral & 375.3 & 334.7 & 386.3 & 274.0 & 553.4 & 156.8 \\
\hline \multirow{3}{*}{ 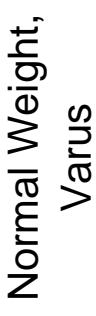 } & medial & 108.2 & 86.5 & 191.2 & 139.4 & 263.1 & 55.6 \\
\hline & lateral & 240.3 & 221.6 & 182.3 & 107.3 & 227.6 & 98.0 \\
\hline & femoral & 380.1 & 312.2 & 377.2 & 261.3 & 536.8 & 158.4 \\
\hline \multirow{3}{*}{ 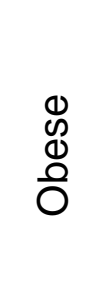 } & medial & 174.3 & 267.2 & 225.4 & 225.4 & 262.6 & 54.9 \\
\hline & lateral & 180.1 & 320.0 & 227.5 & 178.6 & 301.0 & 136.9 \\
\hline & femoral & 348.6 & 593.4 & 451.9 & 406.0 & 544.8 & 191.9 \\
\hline \multirow{3}{*}{ 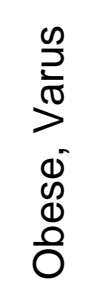 } & medial & 155.2 & 236.4 & 211.3 & 197.9 & 254.3 & 55.7 \\
\hline & lateral & 146.6 & 335.9 & 215.6 & 166.2 & 289.0 & 144.6 \\
\hline & femoral & 324.7 & 592.6 & 432.6 & 381.5 & 535.2 & 193.3 \\
\hline
\end{tabular}

s some consistent patterns observed in the medial and lateral contact areas for normal weight and obese loading conditions. Contact area was usually greater on the lateral cartilage in comparison to the medial regardless of body weight or alignment. At $50 \%$ stance, however, medial contact area was greater than lateral contact area for all four subject types. 
The addition of varus malalignment to normal weight loading increased contact area on the medial cartilage, but did not consistently increase the lateral cartilage contact area. In contrast, both medial and lateral contact area tended to increase due to varus malalignment for the obese loading condition. From 0\%$50 \%$ stance, $\mathrm{OB}$ and $\mathrm{OB}, \mathrm{V}$ medial contact areas were considerably greater than $\mathrm{NW}$ and $\mathrm{NW}, \mathrm{V}$ medial contact areas. $\mathrm{OB}$ and $\mathrm{OB}, \mathrm{V}$ lateral contact areas were also greater than NW and NW,V lateral contact areas between $5 \%$ and $100 \%$ of stance.

Femoral cartilage contact area tended to decrease due to varus malalignment regardless of body weight. However, both $\mathrm{NW}, \mathrm{V}$ and $\mathrm{OB}, \mathrm{V}$ femoral contact area increased at toe-off relative to NW and OB. Increasing body weight produced greater femoral contact area at $25 \%-75 \%$ stance and toe-off regardless of neutral or varus knee alignment. In general, femoral contact area was greatest for OB and least for NW,V. 


\section{CHAPTER 4}

\section{DISCUSSION}

\subsection{Tibiofemoral FE Model}

The FE model results indicated that obesity and varus malalignment both tended to increase contact pressure in the tibiofemoral compartment, but whether these increases in pressure actually lead to OA development is still to be determined. Data from instrumented prosthetic hip joints suggest that articular cartilage can sustain loads of 5-8 MPa through the course of daily activities, with maximum static values recorded as high as $18 \mathrm{MPa}$ [56]. Furthermore, the experimental values listed in Table 10 indicate that articular cartilage in the tibiofemoral compartment would be routinely exposed to pressure in the range of 2-6 MPa. The maximum contact pressure recorded from the normal weight neutral aligned FE model ranged from 2.1-8.3 $\mathrm{MPa}$, which falls within the experimental ranges supported by the literature for normal joint loading. Therefore, the normal weight neutral aligned FE model results can provide a basis for which to compare the effects of varus malalignment and obesity.

Effort has been made to quantify the threshold level of stress leading to cartilage degeneration and $\mathrm{OA}$ development through in vitro studies of cartilage loading. Cell death and cartilage proteoglycan concentration during static and cyclic loading are commonly used markers to investigate the pathological onset of cartilage degeneration. A review of the literature suggests that cartilage cell death and cartilage surface damage occurs at a lower threshold of $14-15 \mathrm{MPa}$ [56-58]. However, cyclic and repetitive loading has the potential to lower this 
range for cell death considerably; significant cell death has been shown to occur in the superficial layer of articular cartilage when exposed to cyclic loads as low at $1 \mathrm{MPa}$ over a span of 14 hours [59].

It was hypothesized that obesity alone would not cause contact stress in the tibiofemoral joint to exceed pathological levels due to gait modifications developed by obese individuals. Obese loading conditions caused greater contact pressure in both the lateral and medial tibiofemoral compartments at most phases of stance, but the majority of the maximum pressure values were safely within the 2-8 MPa range of a normal weight individual and well under the 14-15 MPa pathological threshold for cell death and cartilage surface damage. However, a maximum contact pressure of $10.3 \mathrm{MPa}$ was recorded at $25 \%$ stance on the medial tibial cartilage, which may be of concern when cyclic loading is considered. Additionally, contour plots and contact area measurements indicated that obese loading conditions exposed a larger area of cartilage to pressure, potentially spreading load to regions of cartilage not conditioned to sustain pressure. Overall, it is very possible that the increase in contact pressure at $25 \%$ stance applied cyclically during routine activities, combined with a greater exposure on the cartilage surface would increase an obese individual's susceptibility to cartilage damage and OA development. For this reason, the FE model results refute the initial hypothesis and further emphasize the significant risk of OA development due to obesity.

It was also speculated that knee malalignment could be identified as a mediating factor between obesity and articular cartilage contact stress in relation 
to the development of knee OA. Varus malalignment in particular was considered a significant factor due to the transmission of load to the medial tibial cartilage, resulting in a greater disproportionate allocation of load across the tibiofemoral compartment. It was hypothesized that excess body weight would increase articular cartilage contact stress, but that the additive effect of varus malalignment would cause greater contact stress in the medial tibiofemoral compartment, but not in the lateral.

The results obtained from the tibiofemoral FE model only half support this hypothesis. Excess load due to obesity did in fact increase contact pressure across the tibiofemoral compartment at nearly every phase of stance, but the effect of varus malalignment was less consistent. Varus malalignment increased medial contact pressure as expected, but lateral contact pressure also increased between $25 \%-75 \%$ stance for both normal weight and obese load conditions. The hypothesis was based on the assumption that varus malalignment would function primarily to redistribute load between compartments, but not to increase loading overall. The contour plots in Appendix A and the contact areas in Table 12 show that varus malalignment produced minor changes in area for both normal weight and obese loading, implying that the lateral contact pressure increase is due to load generation rather than area reduction. These results suggest that varus malaligned individuals could be more susceptible to OA development in both tibiofemoral compartments due to the overall increase in joint contact pressure. Despite the increase in lateral contact pressure, the medial articular cartilage in 
varus malaligned knees would likely be more susceptible to OA due to the greater magnitude of medial pressure.

\subsection{Future Work}

The following sections outline weaknesses in the tibiofemoral FE model and discuss future directions for model improvement.

\subsubsection{Damping Factor}

As previously mentioned in Section 2.3.6 of Methods, the extremely large ratio of viscous damping energy to total strain energy is of major concern and will need to be addressed in future generations of the tibiofemoral FE model. Manually prescribing a constant damping factor was seen as a way to control the instabilities in the model and allow the solver to obtain a converged solution; however, the energy ratio indicates that the specified damping factor of 0.002 was too large, resulting in an inaccurate solution [52]. Furthermore, the damping factor should not affect the model solution, but should simply allow the model to converge to its correct solution. A simple analysis was performed to test this theory by loading the tibiofemoral FE model with an $1800 \mathrm{~N}$ load at $0^{\circ}$ flexion and allowing frontal plane rotation of the femur with all other degrees of freedom constrained. The results in Figure 12 clearly show a strong linear relationship between maximum contact pressure and the prescribed damping factor, indicating that the model solution is not independent of damping factor at the range analyzed.

It may still be possible to use a damping factor to control the tibiofemoral FE model instabilities, but it is recommended that adaptive automatic stabilization 
be used in conjunction rather than specifying a constant damping factor. The benefit to this approach is that it allows the Abaqus solver to recalculate the damping factor at each iteration in order to maintain a more appropriate viscous energy ratio. Alternatively, the instabilities in the model may be overcome by introducing small amount of friction at the articulating joint surface in order to control the amount of motion of the model. It is likely that a combination of the two methods may be necessary to control the model instabilities, and it will likely require an iterative process to determine the best overall approach.

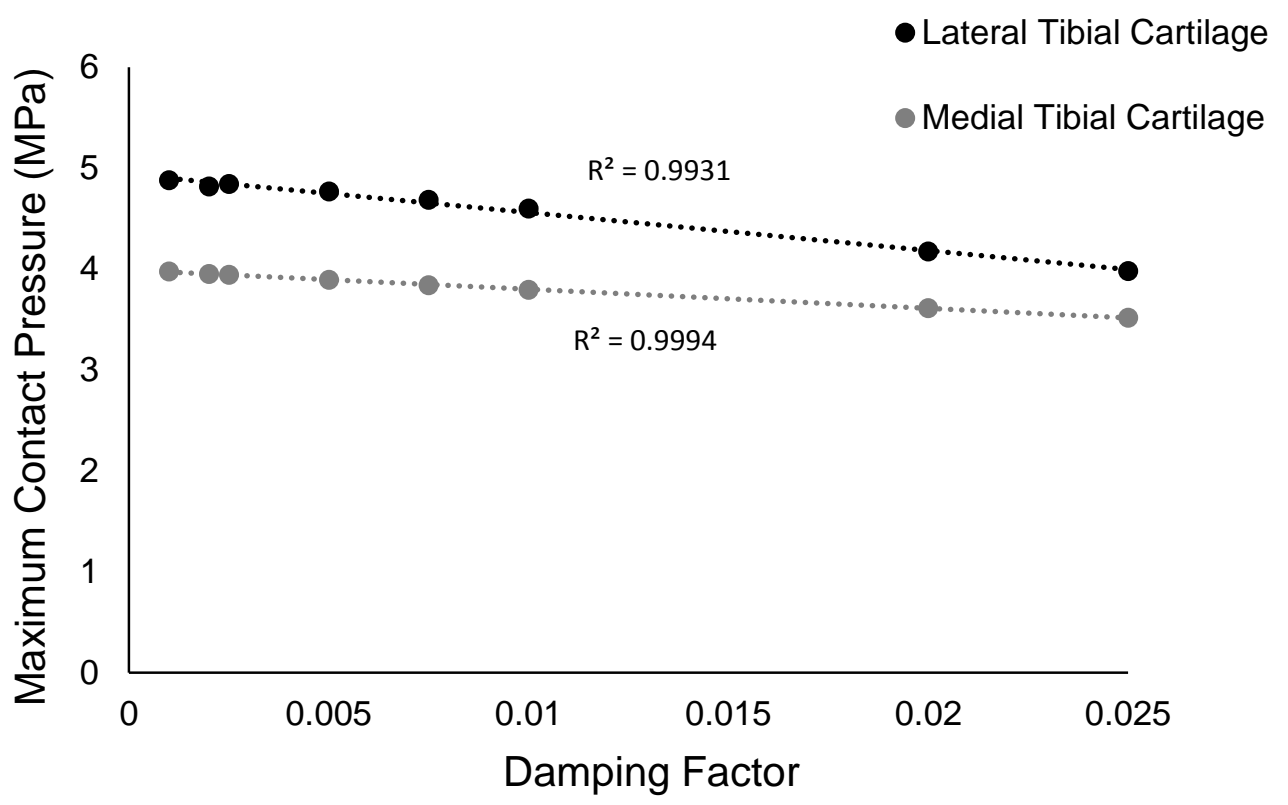

Figure 12: The plot of medial and later tibial cartilage contact pressure as a function of damping factor.

\subsubsection{Material Models}

The material models used in this analysis were very simplistic considering the extreme complexity of biological soft tissue. Adapting the ligament and 
cartilage material models to more realistically capture their physiological properties could increase the accuracy of the model. For example, the ligaments in the tibiofemoral FE model would frequently buckle because the material properties were identical in tension as in compression. In reality, the parallel, regular organization of collagen fibrils in ligaments provide significant strength in tension, but not compression [60]. Water retained by glycsosaminoglicans does provide compressive strength to the ligaments, but a more accurate material model would define the ligament material properties stronger in tension than in compression [60]. The articular cartilage material model could also be improved to better capture the complete response of the tissue. Linear constitutive material models have been shown to produce equivalent contact pressure values in FE analysis as a poromechanical model with fluid pressurization; however, the two approaches produced very different deformation patterns on the cartilage surface [61]. The displacement of the tissue under compression is influenced by the high effective modulus in the linear model, resulting in more rigid motion of the cartilage [61]. An alternative approach could involve depth-dependent material properties, which would better capture the variation in stiffness between the superficial and middle to deep layers of articular cartilage.

Additionally, it may be desirable to model the articular cartilage with different material properties for normal weight and obese subjects. Research suggests that there may be a biochemical or metabolic factors contributing to the development of obesity related OA. Obesity is characterized by the abnormal expression of adipokines, which can bind to the cellular receptors of 
chondrocytes and osteoblasts and lead to break down and remodeling of joint tissue [7]. Therefore, future attempts at comparing normal weigh and obese individuals may want to consider body weight specific material models of articular cartilage.

\subsubsection{Patellofemoral Joint}

The lack of a patellofemoral joint in the FE model may be a significant source of error due to the missing kinetic influence of the patella-femur contact. The joint reaction forces and moments calculated in OpenSim were produced by assuming that the patella acts purely as a kinematic constraint during gait, that is, the loads applied to the femur FE model do not include the contact force of the patella. Research suggests that the patella contact force may become very large during early stance and can reach as high as $265 \mathrm{~N}$ [62]. A simple analysis was performed with the FE model to test the effect of the patella contact force at $0 \%$ stance. Loads and boundary conditions corresponding to a normal weight individual listed in Table 5 were applied to the model with the addition of a compressive force of $65 \mathrm{~N}$ acting in the anterior-posterior direction [62]. The force was applied to the anterior surface of the femur just above the femoral cartilage, which is a rough approximation of the location of the patella at $0 \%$ of stance. The addition of this pseudo patella force decreased the maximum contact pressure by $23 \%$ on the medial tibial cartilage and only $7 \%$ on the lateral tibial cartilage.

Clearly, the patellofemoral interaction has implications on tibiofemoral joint contact pressure, which will have to be accounted for in some way in future efforts. 


\section{CHAPTER 5}

\section{CONCLUSION}

On the whole, the results presented satisfactorily meet the short term objectives of this thesis. A FE model of the tibiofemoral joint was successfully developed and validated against experimental values of joint contact pressure reported in published studies. Furthermore, the FE model was implemented in the Abaqus solver with great effect to produce values of articular cartilage contact pressure within a realistic range for human gait. It was also shown that the joint kinematics and kinetics of obese and malaligned individuals can be modeled using appropriate loading and boundary conditions to predict changes in joint contact pressure due to these factors.

It was hypothesized that obese gait modifications would manifest in joint kinematics, resulting in FE model contact pressure values that would fall within a safe, non-pathological range. Although this hypothesis was disproved, the results were likely influenced by the fact that gait data was collected from obese individuals at $1.25 \mathrm{~m} / \mathrm{s}$ rather than a self-selected walking speed. Gait analysis research has shown that obese individuals walk at a preferred speed of $1.1 \mathrm{~m} / \mathrm{s}$ and that walking slower will reduce joint loads at the knee [11], [12]. It is possible that if this FE study was repeated using kinetic and kinematic data from participants at their self-selected walking speed, then the difference in magnitude of contact pressure between normal weight and obese individuals may decrease.

As a qualitative tool, the FE model functioned well in highlighting changes in joint contact pressure due to the addition of obesity or varus malalignment. In 
order to meet the long term goals of this project and implement the FE model in a clinical application, further work must be done to increase confidence in the quantitative outputs of the model. Addressing the proposed changes outlined in Section 4.2 will greatly increase the FE model's accuracy and applicability for clinical studies focusing on the prevention and treatment of OA. 


\section{WORKS CITED}

[1] "Osteoarthritis Fact Sheet," Arthritis Foundation, January 2008. [Online]. Available: <http://www.arthritis.org/files/images/newsroom/>. [Accessed July 2014].

[2] M. Fransen, L. Bridgett, L. March, D. Hoy, E. Penserga and P. Brooks, "The Epidemiology of Osteoarthritis in Asia," International Journal of Rheumatic Diseases, vol. 14, no. 2, pp. 113-121, 2011.

[3] "Chronic Rheumatic Conditions," World Health Organization, [Online]. Available: <http://www.who.int/chp/topics/rheumatic/en/>. [Accessed July 2014].

[4] H. Gudbergsen, M. Boesen, L. S. Lohmander, R. Christensen, M. Henriksen, E. M. Bartels, P. Christensen, L. Rindel, J. Aaboe, B. Danneskiold-Samsoe, B. F. Riecke and H. Bliddal, "Weight Loss is Effective for Symptomatic Relief in Obese Subjects with Knee OA Independently of Joint Damage Severity Assessed by high-field MRI and Radiography," Osteoarthritis and Cartilage, vol. 20, no. 6, pp. 495502, 2012.

[5] N. Arden and M. C. Nevitt, "Osteoarthritis: Epidemiology," Best Practice \& Research Clinical Rheumatology, vol. 20, no. 1, pp. 3-25, 2006.

[6] R. Moyer, T. Birmingham, B. Chesworth, C. Kean and Griffin J.R., "Alignment, Body Mass, and Their Interaction on Dynamic Knee Joint Load in Patients with Knee O," Osteoarthritis and Cartilage, vol. 18, no. 7, pp. 888-893, 2010.

[7] L. K. King, L. March and A. Anandacoomarasamy, "Obesity and Osteoarthritis," Indian Journal of Medial Research, vol. 138, no. 2, pp. 185-193, 2013.

[8] S. P. Messier, D. J. Gutekunst, C. Davis and P. DeVita, "Weight Loss Reduces Knee-Joint Loads in Overweight and Obese Older Adults with Knee Osteoarthritis," Arthritis \& Rheumatism, vol. 52, no. 7, pp. 20262032, 2005.

[9] D. T. Felson, J. Goggins, J. Niu, Y. Zhang and D. J. Hunter, "The Effect of Body Weight on Progression of Knee Osteoarthritis is Dependent on Alignment," Arthritis and Rheumatism, vol. 50, no. 12, pp. 3904-3909, 2004.

[10] L. Sharma, C. Lou, S. Cahue and D. D. Dunlop, "The Mechanism of the Effect of Obesity in Knee Osteoarthritis: The Mediating Role of 
Malalignment," Arthritis \& Rheumatism, vol. 43, no. 3, pp. 568-575, 2000.

[11] R. C. Browning and R. Kram, "Effects of Obesity on the Biomechanics of Walking at Different Speeds," Medicine \& Science in Sports \& Exercise, vol. 39, no. 9, pp. 1632-1641, 2007.

[12] P. P. Lai, A. K. Leung, A. N. Li and M. Zhang, "Three-Dimensional Gait Analysis of Obese Adults," Clinical Biomechanics, vol. 23, no. 1, pp. S2-S6, 2008.

[13] T. P. Andriacchi, S. Koo and S. F. Scanlan, "Gait Mechanics Influence Healthy Cartilage Morphology and Osteoarthritis of the Knee," The Journal of Bone \& Joint Surgery, vol. 91, no. 1, pp. 95-101, 2009.

[14] J. Arokoski, J. Jurvelin, U. Vaatainen and H. Helminen, "Normal and Pathological Adaptations of Articular Cartilage to Joint Loading," Scandinavian Journal of Medicine \& Science in Sports, vol. 10, no. 4, pp. 186-198, 2000.

[15] J. Runhaar, B. W. Koes, S. Clockaerts and S. Bierna-Zerinstra, "A Systemic Review on Changed Biomechanics of Lower Extremities in Obese Individuals: A Possible Role in Development of Osteoarthritis. Obesity Reviews," Obesity Reviews, vol. 12, no. 12, pp. 1071-1082, 2011.

[16] F. Eckstein, W. Wirth, M. Hudelmaier, V. Stein, V. Lengfelder, S. Cahue, M. Marshall, P. Prasad and L. Sharma, "Patterns of Femorotibial Cartilage Loss in Knees with Neutral, Varus, and Valgus Alignment," Arthritis Care \& Research, vol. 59, no. 11, pp. 1563-1570, 2008.

[17] N. A. Segal, H. J. Yack and P. Khole, "Weight, Rather than Obesity Distribution, Explains Peak External Knee Adduction Moment During Level Gait," American Journal of Physical Medicine \& Rehabilitation, vol. 88, no. 3, pp. 180-246, 2009.

[18] C. Richards and J. Higgins, "Knee Contact Force in Subjects with Symmetrical OA Grades: Differences Between OA Severities," Journal of Biomechanics, vol. 43, no. 13, pp. 2595-2600, 2010.

[19] D. J. Haight, Z. F. Lerner, W. J. Board and R. C. Browning, "A Comparison of Slow, Uphill and Fast, Level Walking on Lower Extremity Biomechanics and Tibiofemoral Joint Loading in Obese and Nonobese Adults," Journal of Orthopaedic Research, vol. 32, no. 2, pp. 324-330, 2014.

[20] Z. Lerner, W. Board and R. Browning, "Effects of Obesity on Lower Extremity Muscle Function During Walking at Two Speeds," Gait \& 
Posture, vol. 39, no. 3, pp. 978-984, 2014.

[21] R. Shirazi and A. Shirazi-Adl, "Computation Biomechanics of Articular Cartilage of Human Knee Joint: Effects of Osteochondral Defect.," Journal of Biomechanics, vol. 42, no. 15, pp. 2458-465, 2009.

[22] M. Adouni and A. Shirazi-Adl, "Evaluation of Knee Joint Muscle Forces an dTissue Stresses-Strains During Gait in Severe OA Versus Normal Subjects," Journal of Orthopaedic Research, vol. 32, no. 1, pp. 69-78, 2014.

[23] E. Pena, J. Pena and M. Doblare, "On Modeling Nonlinear Viscoelsatic Effects in Ligaments," Journal of Biomechanics, vol. 41, no. 12, pp. 265-2666, 2008.

[24] "Q Angle," Physiopedia, [Online]. Available: http://www.physiopedia.com/\%27Q\%27_Angle. [Accessed 2 May 2015].

[25] Materialise, "Mimics Student Edition Course Book v13.1," Materialise, [Online]. Available:

http://mechanical.mines.edu/courses/eggn536/Documents/MimicsSEC ourseBook13.1.pdf. [Accessed 25 July 2015].

[26] R. LaPrade, T. Ly and F. Wentorf, "The posterolateral attachments of the knee: a qualitative and quantitative morphologic analysis of the fibular colalteral ligament, popliteus tendon, popliteofibular ligament, and lateral gastrocnemius tendon," American Journal of Sports Medicine, vol. 31, no. 6, pp. 854-860, 2003.

[27] B. Meister, S. Michael and R. Moyer, "Anatomy and kinematics of the lateral collateral ligament of the knee," American Journal of Sports Medicine, vol. 28, no. 6, pp. 869-878, 2000.

[28] J. Brinkman, P. Schwering and L. Blankevoort, "The insertion geometry of the posterolateral corner of the knee," Journal of Bone and Joint Surgery, vol. 87, pp. 1364-1368, 2005.

[29] R. LaPrade, A. Engebretsen and T. Ly, "The Anatomy of the Medial Part of the Knee," The Journal of Bone and Joint Surgery, vol. 89, no. 9, pp. 2000-2010, 2007.

[30] F. Liu, B. Yue and H. R. Gadikota, "Morphology of the Medial Collateral Ligament of the Knee," Journal of Orthopedic Surgery and Research, vol. 5, no. 69, pp. 1-8, 2010.

[31] J. R. Robinson, A. M. Bull and A. A. Amis, "Structural properties of the medial collateral ligament complex of the human knee," Journal of Biomechanics, vol. 38, no. 5, pp. 1067-1074, 2005. 
[32] R. Siebold, T. Ellert and S. Metz, "Femoral Insertions of the Anteromedial and Posterolateral bundles of the Anterior Cruciate Ligament: Morphology adn arthroscopic Orientation Model for Double-Bundle Bone Tunnel Placement-- a Cadaver Study," Arthroscopy, vol. 24, no. 5, pp. 585-592, 2008.

[33] R. Siebold, T. Ellert and S. Metz, "Tibial Insertions of the Anteromedial and Posterolateral bundles of the Anterior Cruciate Ligament: Morphology, Arthroscopic Landmakes, and Orientation model for Bone Tunnel Placement," Arthoroscopy, vol. 24, no. 2, pp. 154-161, 2008.

[34] R. Shirazi, A. Shirazi-Adl and M. Hurtig, "Role of Cartilage Collagen Fibrils Networks in Knee Joint Biomechanics Under Compression," Journal of Biomechanics, vol. 41, no. 16, pp. 3340-3348, 2008.

[35] G. Limbert, M. Taylor and J. Middleton, "Three-Dimensional Finite Element Modelling of the Human ACL: Simulation of Passive Knee Flexion with a Stressed and Stress-Free ACL," Journal of Biomechanics, vol. 37, no. 11, pp. 1723-1731, 2004.

[36] B. Zielinska and T. L. Hau Donahue, "3D Finite Element Model of Meniscectomy: Changes in Joint Contact Behavior," Journal of Biomechanical Engineering, vol. 128, no. 1, pp. 115-123, 2005.

[37] J. A. Weiss, B. N. Maker and S. Govindjee, "Finite Element Implementation of Incompressible, Transversely Isotropic Hyperelasticity," Computer Methods in Applied Mechanics and Engineering, vol. 135, no. 1-2, pp. 107-128, 1996.

[38] W. Wilson, J. Huyghe and C. van Donkelaar, "Depth-Dependent Compressive Equilibrium Properties of Articular Cartilage Explained by its Composition," Biomechanics and Modeling in Mechanobiology, vol. 6, no. 1-2, pp. 43-53, 2007.

[39] S. Elhamian, M. Alizadeh, M. Shokrieh, A. Karimi and S. Madani, "The Effect of Collagen Fiber Volume Fraction on the Mechanical Properties of Articular Cartilage by Micromechanics Models," Perfusion, p. doi: 10.1177/0267659114547942, 2014.

[40] Y. Dabiri and L. Li, "Influences of the Depth-Dependent Material Inhomogeneity of Articular Cartilage on the Fluid Pressurization in the Human Knee," Medical Engineering \& Physics, vol. 35, no. 11, pp. 1591-1598, 2013.

[41] G. A. Ateshian, B. J. Ellis and J. A. Weiss, "Equivalence Between ShortTime Biphasic and Incompressible Elastic Material Responses," 
Journal of Biomechanical Engineering, vol. 129, no. 3, pp. 405-412, 2006.

[42] M. Kazemi, Y. Dabiri and L. P. Li, "Recent Advances in Computational Mechanics of the Human Knee Joint," Computational and MAthematical Methods in Medicine, vol. 2013, pp. 1-27, 2013.

[43] M. Bendjaballah, A. Shirzai-Adl and D. Zukor, "Finite Element Analysis of Human Knee Joint in Varus-Valgus," Clinical Biomechanics, vol. 12, no. 3, pp. 139-148, 1997.

[44] B. L. Wong, W. C. Bae, J. Chun, K. R. Gratz, M. Lotz and R. L. Sah, "Biomechanics of Cartilage Articulation: Effects of Lubrication and Degeneration on Shear Deformation," Arthritis \& Rheumatology, vol. 58, no. 7, pp. 2065-2074, 2008.

[45] K. N. Hauch, D. F. Villegas and T. L. Haut Donahue, "Geometry, TimeDependent and Failure Properties of Human Meniscal Attachments," Journal of Biomechanics, vol. 43, no. 3, pp. 463-468, 2010.

[46] F. R. Noyes and E. S. Grood, "The Strength of the Anterior Cruciate Ligament in Humans and Rhesus Monkeys," Journal of Bone and Joint Surgery, vol. 58, no. 8, pp. 1074-1082, 1976.

[47] N. Chandrashekar, H. Mansouri and J. Slauterbeck, "Sex-Based Differences in the Tensile Properties of of the Human Anterior Cruciate Ligament," Journal of Biomechanics, vol. 25, no. 5, pp. 511-518, 1992.

[48] J. Hicks, "Joint Reaction Analysis," SimTK, 1 January 2014. [Online]. Available: $<$ http://simtkconfluence.stanford.edu:8080/display/OpenSim/Joint Reactions Analysis>. [Accessed April 2015].

[49] "Sample Size Calculators," DSS Research, [Online]. Available: https://www.dssresearch.com/KnowledgeCenter/toolkitcalculators/sam plesizecalculators.aspx.. [Accessed 23 February 2015].

[50] J. Dunne and C. Au, "Gait 2392 and 2354 Models," SimTK, 9 August 2013. [Online]. Available:

<http://simtkconfluence.stanford.edu:8080/display/OpenSim/Gait+2392 +and+2354+Models>. [Accessed 2015 April 30].

[51] S. Wangerin, "Development and validation of a human knee joint finite element model for tissue stress and strain predictions during exercise," California Polytechnic State University, San Luis Obispo, 2013.

[52] "Abaqus Analysis User's Manual Version 6.7," Dassault Systemes, [Online]. Available: 
http://www.egr.msu.edu/software/abaqus/Documentation/docs/v6.7/bo oks/usb/default.htm?startat=book01.html. [Accessed 4 May 2015].

[53] A. M. Seitz, A. Lubomierski, B. Friemert, A. Ignatius and L. Durselen, "Effect of Partial Meniscectomy at the Medial Posterior Horn on Tibiofemoral Contact Mechanics and Meniscal Hoop Strains in Human Knees," Journal of Orthopaedic Research, vol. 30, no. 6, pp. 934-942, 2012.

[54] J. M. Marzo and J. Gurske-DePerio, "Effects of Medial Meniscus Posterior Horn Avulsion and Repair on Tibiofemoral Contact Area and Peak Contact Pressure with Clinical Implications," The American Journal of Sports Medicine, vol. 37, no. 1, pp. 124-129, 2009.

[55] Y. Morimoto, M. Ferretti, M. Ekdahl, P. Smolinksi and F. H. Fu, "Tibiofemoral Joint Contact Area and Pressure After Single- and Double-Bundle Anterior Cruciate Ligament Reconstruction," The Journal of Arthroscopic and Related Surgery, vol. 25, no. 1, pp. 62-69, 2009.

[56] K. M. Clements, Z. C. Bee, G. V. Crossingham, M. A. Adams and M. Sharif, "How severe must repetitive loading be to kill chondrocytes in articular," Osteoartritis and Cartilage, vol. 9, no. 5, pp. 499-507, 2001.

[57] D. D. D'Lima, S. Hashinmoto, P. C. Chen, C. W. Colwell and M. K. Lotz, "Human chondrocyte apoptosis in response to mechanical injury," Journal of the OsteoArthritis Research Society International, vol. 9, no. 8, pp. 712-719, 2001.

[58] P. A. Torzilli, R. Grigiene, J. Borrelli and D. L. Helfet, "Effect of Impact Load on Articular Cartilage: Cell Metabolism and Viability, and Matrix Water Content," Journal of Biomechanical Engineering, vol. 121, no. 5, pp. 433-441, 1999.

[59] E. Lucchinetti, C. S. Adams, W. E. Horton and P. A. Torzilli, "Cartilage viability after repetitive loading: a preliminary report," Osteoarthritis and Cartilage, vol. 10, no. 1, pp. 71-81, 2002.

[60] V. B. Duthon, C. Barea, S. Abrassart, J. H. Fasel, D. Fritschy and J. Menetrey, "Anatomy of the Anterior Cructiate Ligament," Knee Surgery, Sports Traumatology, Arthroscopy, vol. 14, no. 3, pp. 204213, 2006.

[61] L. P. Li and K. B. Gu, "Reconsideration on the Use of Elastic Models of Predict the Instantaneous Load REsponse of the Knee Joint," Proceedings of the Institution of Mechanical Engineers, Part H: Journal of Engineering in Medicine, vol. 225, no. 9, pp. 888-896, 2011. 
[62] Y.-C. Lin, J. P. Walter, S. A. Banks, M. G. Pandy and B. J. Fregly, "Simultaneous prediction of muscle and contact forces in the knee during gait," Journal of Biomechanics, vol. 43, no. 5, pp. 945-952, 2010. 


\section{APPENDICES}

Appendix A: Contour Plots of Contact Pressure on the Surface of the Tibial and Femoral Articular Cartilages

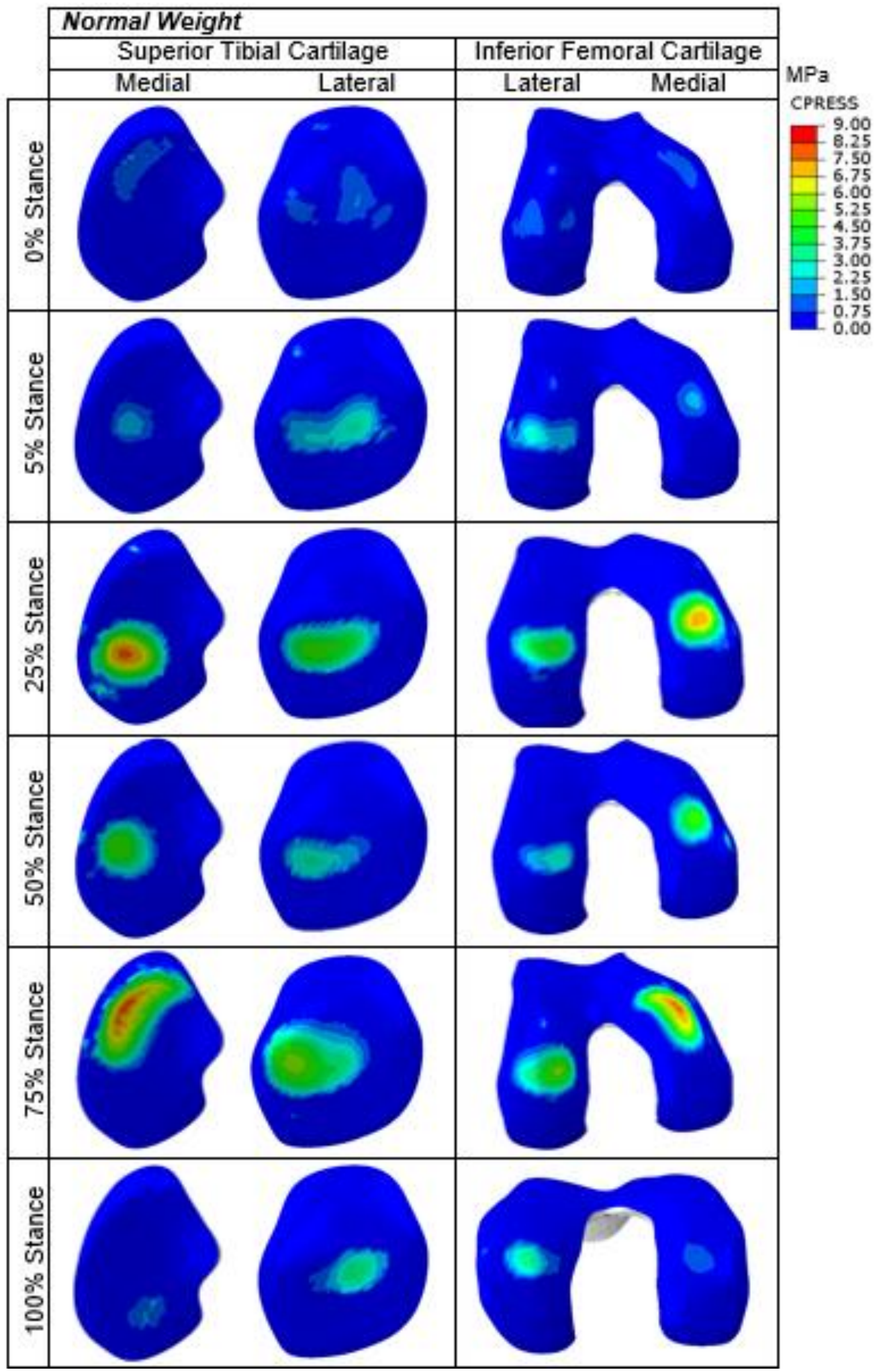




$$
\begin{aligned}
& 001 \\
& 800 \\
& 800 \\
& 800 \\
& 800 \\
& 000
\end{aligned}
$$




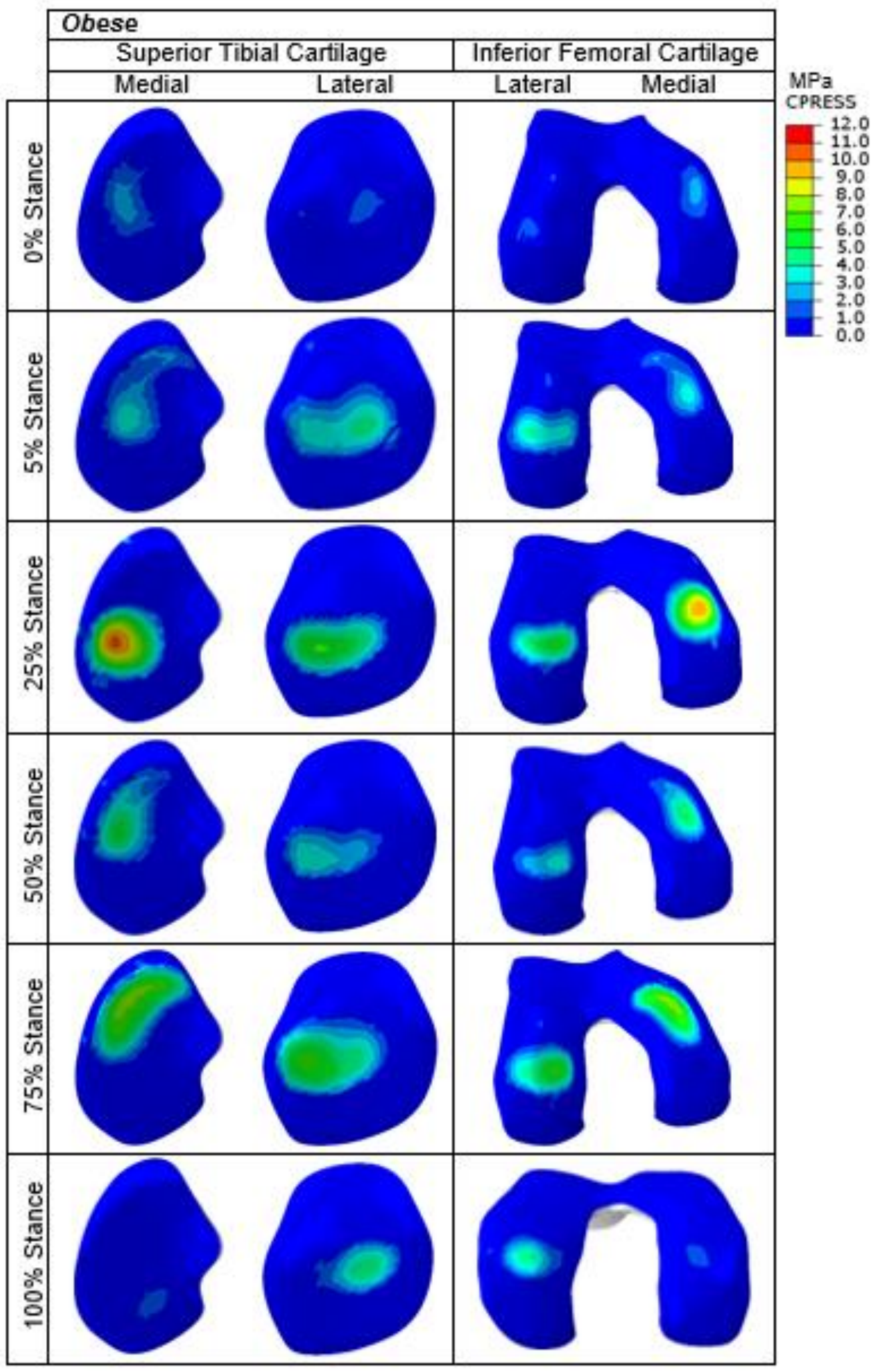




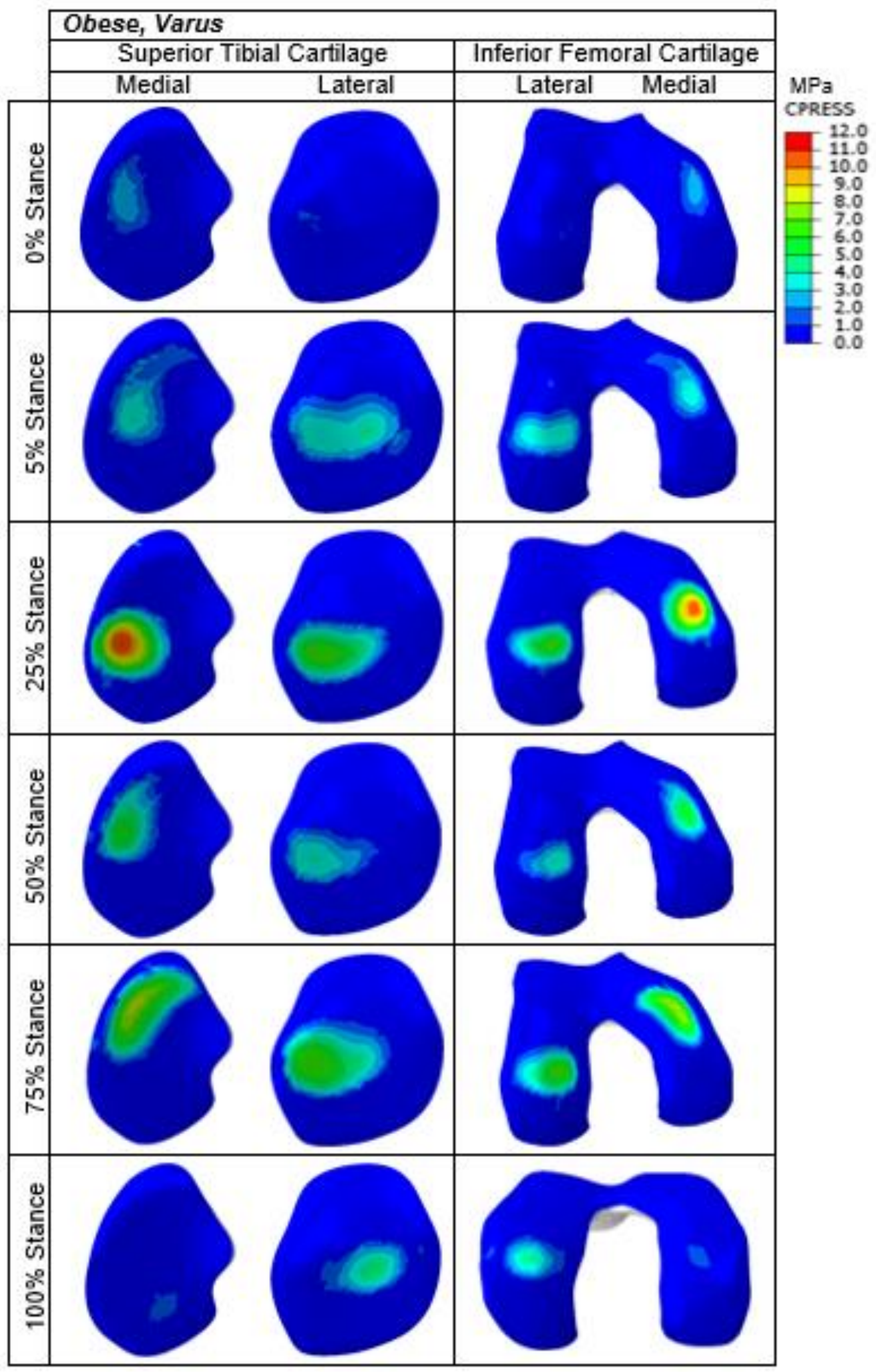

